\title{
The partial linear model in high dimensions
}

\author{
High-dimensional partial linear model
}

\author{
Patric Müller and Sara van de Geer \\ Seminar für Statistik, ETH Zurich
}




\begin{abstract}
Partial linear models have been widely used as flexible method for modelling linear components in conjunction with non-parametric ones. Despite the presence of the non-parametric part, the linear, parametric part can under certain conditions be estimated with parametric rate. In this paper, we consider a high-dimensional linear part. We show that it can be estimated with oracle rates, using the LASSO penalty for the linear part and a smoothness penalty for the nonparametric part.
\end{abstract}

Key words: doubly penalised lasso, high-dimensional partial linear model, lasso, nuisance function, semi-parametric model, variable selection

\title{
1 Introduction
}

Consider the partial linear model where the expectation of the response variable $Y$ depends on two predictors $(X, Z)$. The dependence of $Y$ on the first explanatory variable $X$ is linear, whether a non-linear, unknown, nuisance function defines the dependence on $Z$.

In order to deal with this model we need some special techniques which take into account its particular properties. Simply neglecting the non-linear part and using the standard least square estimator does not lead to the correct answers, on the other hand directly applying some non-linear regression (i.e. treating the linear part as non-linear) is often too rough.

The partial linear model was studied extensively, see e.g. Green and Yandell (1985), Wahba (1990) and more recently Härdle and Liang (2007) and the references therein. In Mammen and van de Geer (1997) the penalised least squares estimator is shown to be consistent. Asymptotic normality for the linear part was also shown. These results are valid in the low dimensional case, where the number of observations $n$ is much larger than the number of variables $p$ in the linear part i.e. $n \gg p$.

The high-dimensional case, where $n \ll p$, is more challenging. The model is now underspecified and the methods proposed in Mammen and van de Geer (1997) can not be directly applied. We need some more techniques in order to overcome these new difficulties.

There is a large body of work on linear high dimensional models. A common approach is to construct penalised estimators like the LASSO (least absolute shrinkage and selection operator proposed in Tibshirani (1996)) or the ridge regression and elastic net (see Zou and Hastie (2005)). The LASSO is widely 
studied (see e.g. Koltchinskii (2011) and Bühlmann and van de Geer (2011) and references therein) and gives remarkable results in sparse contexts.

In this paper we study the high-dimensional partial linear model. Our main contribution is to combine the methods used in Mammen and van de Geer (1997) for the low dimensional case with the standard LASSO and obtain an estimator that can deal with and overcome both extra difficulties given from the high-dimensional and the non-linear part of the model.

We prove theoretical results giving bounds for both the prediction and the estimation error. In the last part of the paper we present simulation results. We compare the performance of our method with the standard LASSO with (un-)known nuisance function.

The paper is organised as follows. We begin in Section 2 with notation and model description. The required conditions are here listed and discussed. Section 3 describes our main results. In Section 4 we present simulations. These confirm the previous theoretical results. Section 6 contains technical tools from empirical process theory and concentration inequalities. These tools are required for the proof of the results from Section 3 , which are also part of Section 6. A set of tables with detailed results of the simulations can be found in Section 7

\section{Notation and model assumption}

In this section we describe more in detail the model we study. After the formal description of the model, we define some extra conditions we need in order to prove our results. For each condition we further add a short comment on their strength. The notation we use is explained in the following subsection.

\section{$2.1 \quad$ Notation}

Through all the paper we always use $i \in\{1, \ldots, n\}$ and $j \in\{1, \ldots, p\}$.

For a vector $\beta \in \mathbb{R}^{p}$ the $\ell_{1}$-norm is $\|\beta\|_{1}:=\sum_{j=1}^{p}\left|\beta_{j}\right|$. For a fixed $\beta^{0} \in \mathbb{R}^{p}$, we denote with $S_{0}$ the set of all non-zero components of $\beta^{0} \in \mathbb{R}^{p}$ and with $s_{0}$ its cardinality. Define for all $\beta \in \mathbb{R}^{p}$, the vector $\beta_{S_{0}} \in \mathbb{R}^{p}$ where $\left(\beta_{S_{0}}\right)_{i}=$ $\left|\operatorname{sign}\left(\beta_{i}^{0}\right)\right| \beta_{i}$, i.e. the components in $S_{0}^{c}$ are set to 0 . Define $\beta_{S_{0}^{c}}:=\beta-\beta_{S_{0}}$.

Let $x \in \mathbb{R}^{p}$ and $z \in \mathbb{R}^{d}$ be high-dimensional and low-dimensional random 
variables, respectively. Denote by $Q$ the distribution of $(x, z)$ and by $Q_{x}\left(Q_{z}\right)$ the marginal distribution of $x(z)$. We let $\left\{\left(x_{i}, z_{i}\right)\right\}_{i=1}^{n}$ be $n$ i.i.d. copies of $(x, z)$. In matrix notation we denote with $X \in M_{n \times p}(\mathbb{R})$ the matrix with rows $x_{i} \in \mathbb{R}^{p}$ $(i=1, \ldots, n)$ and columns $X_{j} \in \mathbb{R}^{n}(j=1, \ldots, p)$. With $X_{i j}$ we denote the $(i, j)^{t h}$ component of $X$. Similarly, we let $Z$ be the $n \times d$-matrix with rows $z_{i}$ $(i=1, \ldots, n)$.

For $f: \mathbb{R}^{p+d} \rightarrow \mathbb{R}$ a measurable function, we define, with some abuse of notation $f(X, Z):=\left(f_{1}, \ldots, f_{n}\right)^{T}:=\left(f\left(x_{1}, z_{1}\right), \ldots, f\left(x_{n}, z_{n}\right)\right)^{T}$. The squared $L_{2}$-norm is $\|f\|^{2}:=\mathbb{E} f^{2}(x, z)$, where $\mathbb{E}$ is the expectation with respect to the distribution $Q$. The sup-norm is $\|f\|_{\infty}:=\sup _{\mathrm{x}, \mathrm{z}}|f(\mathrm{x}, \mathrm{z})|$ and the squared empirical norm is defined as

$$
\|f\|_{n}^{2}:=\frac{1}{n} \sum_{i=1}^{n} f^{2}\left(x_{i}, z_{i}\right) .
$$

More generally, for a vector $v \in \mathbb{R}^{n}$, we write $\|v\|_{n}^{2}:=v^{T} v / n$.

\subsection{Model description and motivation}

Let $\mathcal{G}$ be a linear subspace of $L_{2}\left(Q_{z}\right), g^{0} \in \mathcal{G}$ and $\beta^{0} \in \mathbb{R}^{p}$ be fixed.

The response variable $y \in \mathbb{R}$ depends linearly on $x$ and in a non-parametric way on $z$. The observed variables are i.i.d. copies of $(x, z, y)$, whereas $\left(\beta^{0}, g^{0}\right)$ are unknown parameters. The resulting model is then

$$
y=x \beta^{0}+g^{0}(z)+e,
$$

where $e$ is the error term which can be interpreted as noise.

The observations are denoted by $\left\{\left(x_{i}, z_{i}, y_{i}\right)\right\}_{i=1}^{n}(n$ i.i.d. copies of $(x, z, y))$. In matrix notation we have

$$
Y=X \beta^{0}+g^{0}(Z)+E \in \mathbb{R}^{n}
$$

where $g^{0}(Z):=\left(g^{0}\left(z_{1}\right), \ldots, g^{0}\left(z_{n}\right)\right)^{T} \in \mathbb{R}^{n}, E:=\left(e_{1}, \ldots, e_{n}\right)^{T} \in \mathbb{R}^{n}$ and $Y:=$ $\left(y_{1}, \ldots, y_{n}\right)^{T} \in \mathbb{R}^{n}$.

Remark 2.1. In this paper we take $p \gg n$. Consequently we have a highdimensional linear model with nuisance function.

Semi-parametric models are quite common in low dimensional contexts. In high-dimensional settings linear models (possibly after suitable coordinate transformation) are widely analysed. In some cases however there is strong evidence to believe that one or some variables have a non-linear influence on the response. 
Consider the following (fictive) example:

Example 2.1. Take $Y$ is the yield of some genetically modified plants; $X$ represents the gene expression data of plants; $Z$ are the factors 'water' and 'temperature'. It is reasonable to assume that $Y$ linearly depends on $X$, but not on $Z$ (too much or few water and too high or low temperature reduce the yield). Model (1) provides a reasonable approach to describe this problem.

\subsection{Estimator and model assumptions}

Let $J(\cdot, \cdot)$ be a (semi-)scalar product on $\mathcal{G}$ and $J(\cdot)$ be the corresponding (semi-)norm. We define the doubly penalised least square estimator $(\hat{\beta}, \hat{g})$ as:

$$
(\hat{\beta}, \hat{g}):=\underset{(\beta, g) \in \mathbb{R}^{p} \times \mathcal{G}}{\arg \min }\left\{\|Y-X \beta-g\|_{n}^{2}+\lambda\|\beta\|_{1}+\mu^{2} J^{2}(g)\right\}
$$

We thus propose a doubly penalised least square estimator. The idea behind it is that the $\ell_{1}$-penalisation on the linear coefficients controls the sparsity of $\hat{\beta}$, whether the second penalty term keeps the estimation of $g$ under control. In our hope our estimator has the desired properties given from each of the two different penalisations and this, possibly, without paying a too high price in terms of prediction and estimation error.

Remark 2.2. If $g^{0}$ is known, the restricted version of (2) with $g=g^{0}$ gives the standard LASSO for the linear model (see $10 p)$.

Remark 2.3. When $z \in \mathbb{R}$ (i.e., $d=1$ ) a common choice for $J^{2}(g)$ is the $L_{2}$-norm of the second derivative, $J^{2}(g)=\int_{\mathbb{R}}\left(g^{\prime \prime}(z)\right)^{2} d \nu(z)$, where $\nu$ is some probability measure (see e.g. Wahba (1990), Green et al. (1994)).

In the following lines we give a set of conditions we assume in this paper. Their strength is also shortly discussed.

Condition 2.1 (Gaussian condition).

The errors $e_{1}, \ldots, e_{n}$ are i.i.d. standard Gaussian random variables independent of $\left\{x_{i}, z_{i}\right\}_{i=1}^{n}$.

Gaussian errors is a quite strong, but not unusual assumption. In any case this condition can be easily relaxed to sub-Gaussian errors. We have for simplicity assumed unit variance for the errors. In practice, the variance of the errors will generally not be know. The tuning parameters then will be scaled by an estimated error standard deviation/variance.

Condition 2.2 (Design condition). 
For some constant $K_{X}$ it holds that

$$
\max _{i, j}\left|X_{i j}\right| \leq K_{X}
$$

A bound on the $X$-values is a quite restrictive assumption. However we can often approximate a non-bounded distribution with its truncated version.

Define

$$
h(Z):=\mathbb{E}[X \mid Z], \quad \tilde{X}:=X-\mathbb{E}[X \mid Z] .
$$

Condition 2.3 (Eigenvalue condition).

The smallest eigenvalue $\Lambda_{\tilde{X}, \min }^{2}$ of $\mathbb{E} \tilde{X}^{T} \tilde{X} / n$ is positive.

Furthermore the largest eigenvalue of $\mathbb{E} h^{T} h / n$, denoted by $\Lambda_{h, \max }^{2}$, is assumed to be finite.

This condition ensures that there is enough information in the data to identify the parameters in the linear part.

Let for each $u>0 N\left(u, \mathcal{G},\|\cdot\|_{\infty}\right)$ be the smallest value of $N$ such that there exists $G$, a subset of $\mathcal{G}$ of cardinality $N$, for which

$$
\sup _{g \in \mathcal{G}} \min _{\tilde{g} \in G}\|g-\tilde{g}\|_{\infty} \leq u
$$

Then $H\left(\cdot, \mathcal{G},\|\cdot\|_{\infty}\right):=\log N\left(\cdot, \mathcal{G},\|\cdot\|_{\infty}\right)$ is called the entropy of $\mathcal{G}$ for the supremum norm.

Condition 2.4 (Entropy condition).

For some constants $A$ and $m>1 / 2$ one has

$$
\mathcal{H}\left(u,\{g:\|g\| \leq 1, J(g) \leq 1\},\|\cdot\|_{\infty}\right) \leq A u^{-1 / m}, u>0 .
$$

This condition summarizes the assumed "smoothness" of the nonparametric part. For example, when $Q_{z}$ has support in $[0,1]$, the choice of Remark 2.3 with $\nu$ Lebesgue measure on $[0,1]$ has $m=2$.

Condition 2.5 (Penalty condition).

For some constant $K_{g}$, it holds that

$$
\sup _{\|g\| \leq 1, J(g) \leq 1}\|g\|_{\infty} \leq K_{g}
$$

This condition states that, if the $L_{2}$ norm of $g$ and $J(g)$ are bounded, then the 
supremum norm of $g$ is bounded as well. This avoids functions with high and very steep peaks.

Condition 2.6 ( $J$ and $h$ condition).

For some constant $J_{h}$, it holds that

$$
J(h) \leq J_{h}
$$

This condition is fulfilled for $J_{h}=0$ if $x$ and $z$ are independent and $J$ is chosen as in Remark 2.3

\section{Results}

In this section we present our main results. We provide theoretical guarantees in term of prediction and estimation for estimator (2). Our first theorem proves the convergence of our method, whether the second leads to an oracle result. In particular Theorem 3.2 shows that, up to a constants, our method performs as good as if the nuisance function $g^{0}$ were known.

We use the short-hand notation

$$
\mathcal{F}:=\left\{f=X \beta+g: g \in \mathcal{G}, \beta \in \mathbb{R}^{p}\right\}
$$

and

$$
f^{0}:=X \beta^{0}+g^{0}
$$

Remark 3.1. (Orthogonal decomposition)

For every $f, f^{0} \in \mathcal{F}, f-f^{0}=\tilde{X}\left(\beta-\beta^{0}\right)+\left[h\left(\beta-\beta^{0}\right)+g-g^{0}\right]$ is an orthogonal decomposition i.e.:

$$
\left\|f-f^{0}\right\|^{2}=\left\|\tilde{X}\left(\beta-\beta^{0}\right)\right\|^{2}+\left\|h\left(\beta-\beta^{0}\right)+g-g^{0}\right\|^{2}
$$

Define now

$$
\tau_{\mu, R}(f):=\tau(f):=\lambda\|\beta\|_{1} /\left(R \sqrt{\delta_{0} / 2}\right)+\sqrt{\|X \beta+g\|^{2}+\mu^{2} J^{2}(g)},
$$

where $\delta_{0}$ is a fixed small constant. Values and optimization of the constants are in this paper of minor interest. However we give hereafter indications and or values for the mentioned constants. Note that $\tau$ is a (semi-)norm. This 
is however not used for our results, we will only use in Lemma 6.1 the cone property $\tau(s f)=s \tau(f)$ for all $f$ and all $s>0$.

Theorem 3.1. Assume Conditions 2.1, 2.2, 2.3, 2.4 and 2.5. Let $\delta_{T}$ be a (small) constant, and $\kappa_{T}$ be a (large) constant, suitably chosen, depending on $\Lambda_{\tilde{X}, \min }, \Lambda_{h, \max }, K_{X}, J_{h}, A$ and $m$ only. Take

$$
\kappa_{T} n^{-\frac{2 m}{2 m+1}} \leq \mu^{2} \leq \frac{\delta_{0} R^{2}}{2\left(1+J\left(g^{0}\right)+J_{h}\right)^{2}} .
$$

Assume $R^{2} \leq \lambda \leq 1$

$$
\begin{gathered}
\frac{4 \lambda^{2} s_{0}}{\Lambda_{\tilde{X}, \min }^{2}} \leq \delta_{0} R^{2}, \\
\lambda \geq \frac{\sqrt{2 \log (2 p) / n}}{\delta_{T}}
\end{gathered}
$$

and

$$
\Lambda:=1+\frac{\Lambda_{h, \max }}{\Lambda_{\tilde{X}, \min }} \leq \mu^{-1}
$$

Then with probability at least $1-3 \exp \left[-n \delta_{T}^{2} \mu^{2}\right]$, it holds that $\tau\left(\hat{f}-f^{0}\right) \leq R$.

Asymptotics To obtain a clearer picture of the result, let us rephrase it in an asymptotic framework, where $1 / \Lambda_{\tilde{X} \text {, min }}, \Lambda_{h, \max }, K_{X}, J_{h}, J\left(g^{0}\right)$ and $A$ are all bounded by a fixed constant and $m$ is fixed. Then one sees that for $\lambda$ and $\mu$ having the usual order $\lambda \asymp \sqrt{\log p / n}$, respectively $\mu \asymp n^{-\frac{m}{2 m+1}}$, and when $s_{0} \log p / n=o\left(n^{-\frac{2 m}{2 m+1}}\right)$ (that is, the oracle rate for the linear part established in Theorem 3.2 is faster than the rate of convergence for estimating the nuisance part) then the overall rate of convergence is $\tau\left(\hat{f}-f^{0}\right)=\mathcal{O}_{\mathbf{P}}\left(n^{-\frac{m}{2 m+1}}\right)$. This in particular implies $\left\|\hat{g}-g^{0}\right\|=\mathcal{O}_{\mathbf{P}}\left(n^{-\frac{m}{2 m+1}}\right)$ and $J(\hat{g})=\mathcal{O}_{\mathbf{P}}(1)$.

Discussion on the constants The results presented in Theorem 3.1 are valid for $\delta_{0}=1 / 253$. $\mu$ and $R$ are intended to be small constants. The constants $\kappa_{T}$ and $\delta_{T}$ are big and small respectively. They can be for example defined along the lines of (35) and (34). We remind that the optimisation of the constants in this paper is of minor interest.

Theorem 3.2. Assume the same conditions of Theorem 3.1 with the constants 
$\delta_{0}$ and $\delta_{T}$ small enough. Then with probability at least $1-(2 / p)+6 \exp \left[-n \delta_{T}^{2} \mu^{2}\right]$

$$
\left\|\tilde{X}\left(\hat{\beta}-\beta^{0}\right)\right\|_{n}^{2}+\lambda\left\|\hat{\beta}-\beta^{0}\right\|_{1} / 4 \leq \frac{s_{0} \lambda^{2}}{\Lambda_{\tilde{X}, \min }^{2}}
$$

Remark 3.2. This theorem contains two important results, we get prediction results

$$
\left\|\tilde{X}\left(\hat{\beta}-\beta^{0}\right)\right\|_{n}^{2} \leq \frac{s_{0} \lambda^{2}}{\Lambda_{\tilde{X}, \min }^{2}}
$$

and estimation results

$$
\left\|\hat{\beta}-\beta^{0}\right\|_{1} \leq \frac{4 s_{0} \lambda}{\Lambda_{\tilde{X}, \min }^{2}} .
$$

Remark 3.3. Theorem 3.2 says that one can estimate $\beta^{0}$ in $\|\cdot\|_{1}$-norm with the same rate as in the case where the nuisance parameter $g^{0}$ is known. In asymptotic terms, with $\lambda \asymp \sqrt{\log p / n}$ and $\mu \asymp n^{-\frac{m}{2 m+1}}$, this rate is $\mathcal{O}_{\mathbf{P}}\left(s_{0} \sqrt{\log p / n}\right)$. One may verify moreover that the theoretical (out of sample) prediction error, $\left\|X\left(\hat{\beta}-\beta^{0}\right)\right\|^{2}$, the empirical prediction (in sample) error $\left\|X\left(\hat{\beta}-\beta^{0}\right)\right\|_{n}^{2}$ and the squared $\ell_{2}$-error $\left\|\hat{\beta}-\beta^{0}\right\|_{2}^{2}$ are all of order $s_{0} \log p / n$ in probability.

\section{Numerical results}

In this section we present the results of a pseudo real data study. We compare the performance of our estimator (2) with the LASSO estimator.

The following model

$$
\tilde{Y}=X \beta^{0}+E
$$

is a simpler version of (1), where $g^{0}$ is known and is the zero function. A widely used estimator in this case is the Lasso (least absolute shrinkage and selection operator) $\hat{\beta}^{\text {lasso }}$, (Tibshirani (1996)). That is:

$$
\hat{\beta}^{\text {lasso }}:=\underset{\beta}{\arg \min }\|Y-X \beta\|_{n}^{2}+\lambda\|\beta\|_{1}
$$

Under some compatibility assumptions (see van de Geer (2007), Koltchinskii (2009a), Koltchinskii (2009b) and Bickel et al. (2009)) we have, with high probability, the following performance for the Lasso:

$$
\left\|X\left(\hat{\beta}^{\text {lasso }}-\beta^{0}\right)\right\|_{n}^{2}+\lambda\left\|\hat{\beta}^{\text {lasso }}-\beta^{0}\right\|_{1} \leq \frac{4 \lambda^{2} s_{0}}{\phi_{0}^{2}},
$$


where $\phi_{0}$ is the so called compatibility constant (see Theorem 6.1 and Corollary 6.2 in Bühlmann and van de Geer (2011)). This result is very similar to Theorem 3.2 which imply that our method should work, asymptotically, as good as the Lasso in the case where the function $g^{0}$ is known.

In this section we will therefore compare our estimator with the Lasso.

\subsection{Dataset and settings}

We construct the pseudo real dataset for Model (1) as follows:

We take $X$ as a $n \times p$ matrix, from real data. $X$ is obtained by randomly picking out $p$ components from one of the following datasets.

- Leukemia, also used in Dettling (2004) and Bühlmann and Mandozzi (2013).

- Prostate, also used in Dettling (2004) and Bühlmann and Mandozzi (2013).

$Z$ is simulated (see Remark 4.1). We furthermore analyse two different cases. We distinguish the case where $X$ and $Z$ are independent or dependent. To create dependency in the active set of $X$ we redefine the values of the first three columns of the matrix $X$ as follows:

$$
X_{1}:=2 Z+V_{1} \quad, \quad X_{2}:=2 Z^{2}+V_{2} \quad X_{3}:=-Z+V_{3},
$$

where the $V_{j}$ are $n$-dimensional vectors with i.i.d normally distributed components. The resulting empirical correlation between $X_{1}$ and $Z$ is on average 0.74 .

We let the active components of $\beta^{0}$ assume values \pm 1 with probability 0.5 each. Without loss of generality we take $S_{0}:=\left\{1, \ldots, s_{0}\right\}$, i.e. the first $s_{0}$ components of $\beta^{0}$ are different from zero.

We let $z$ be of dimension $d:=1$. Consequently $Z \in \mathbb{R}^{n}$.

Remark 4.1. In general it is (almost) impossible to properly estimate a real function from observational data in a region where there are no or very few observations. In order to avoid big gaps in $Z$ (intervals with few or none observations), we keep $z_{i}$ i.i.d. copies of $z \sim U n i f[-0.5,0.5]$. Observe that actually gaps do not cause very big problems in term of prediction. Only the estimation of $g$ could be imprecise in intervals with very few observations.

In any case, an appropriate prior standard transformation can be applied in order to make our data "look better". 
The semi-real data are then generated as

$$
Y=X \beta^{0}+g^{0}(Z)+\varepsilon
$$

We compare the following three estimators:

- Lasso with known function $g^{0}(\mathrm{LK})$ :

$$
\hat{\beta}^{\text {lasso } g^{0}}:=\underset{\beta}{\arg \min }\left\|Y-g^{0}(z)-X \beta\right\|_{n}^{2}+\lambda\|\beta\|_{1}
$$

One can quickly notice that this is nothing else than Lasso for the linear high-dimensional model.

- Standard Lasso (LN):

$$
\hat{\beta}^{\text {lasso }}:=\underset{\beta}{\arg \min }\|Y-X \beta\|_{n}^{2}+\lambda\|\beta\|_{1}
$$

- Our estimator, the Doubly Penalised Lasso (DP):

$$
(\hat{\beta}, \hat{g}):=\underset{(\beta, g) \in \mathbb{R}^{p} \times \mathcal{G}}{\arg \min }\left\{\|Y-X \beta-g\|_{n}^{2}+\lambda\|\beta\|_{1}+\mu^{2} J^{2}(g)\right\}
$$

For each one of the datasets and each estimator we fit 36 designs with 1000 repetitions each. The designs are obtained by varying the following parameters:

- The dimension $p$, or number of variables in the model. In each simulation run, $p$ covariables are chosen at random among all covariables in the dataset. We take $p$ either 250 or 1000 .

- The sparsity $s_{0}$. This denotes the number of non-zero components of $\beta_{0}$. We let $s_{0}$ be 5 or 15 .

- The function $g^{0}(z)$. We use the following three nuisance functions (see Figure 1).

$$
\begin{gathered}
g_{1}^{0}(z):=0 \\
g_{2}^{0}(z):=-20 z^{2}-10 \\
g_{3}^{0}(z):=3\left(e^{2 z}+\sin (12 z)\right)
\end{gathered}
$$

Our intention is to have a 'representative' sample among the bounded functions. Therefore we choose an "easy" quadratic function $\left(g_{2}\right)$ and a more complicated one $\left(g_{3}\right)$. In order to give importance to both the linear and the non-linear part of the design the functions $g_{2}$ and $g_{3}$ have 


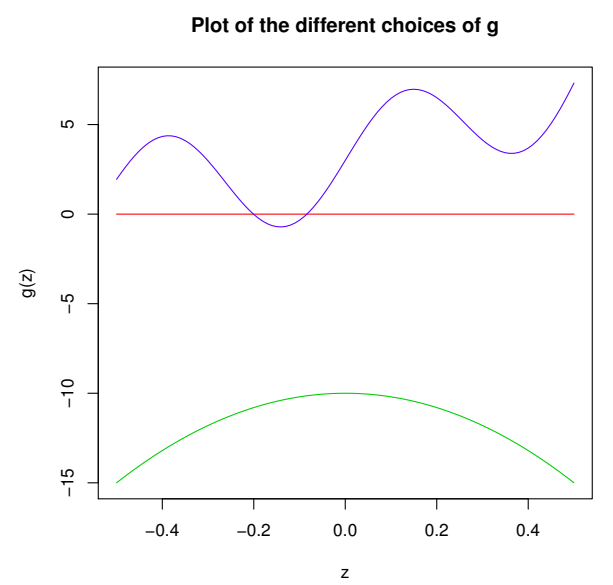

Figure 1: The three functions used for testing the methods: $g_{1}(z) \equiv 0, g_{2}(z)=$ $-20 z^{2}-10$ and $g_{3}(z)=3\left(e^{2 z}+\sin (12 z)\right)$

comparable range of values as $X \beta^{0}$.

The trivial case where there is no nuisance function is represented by $g_{1}^{0}$.

- The linear signal to noise ratio (ISNR), defined as

$$
\operatorname{lSNR}:=\sqrt{\frac{\left\|X \beta^{0}\right\|_{n}^{2}}{\sigma^{2}}}
$$

The lSNR can be 2,8 or 32 .

Remark 4.2. We furthermore define the total signal to noise ratio:

$$
\operatorname{tSNR}:=\sqrt{\frac{\left\|X \beta^{0}+g^{0}\right\|_{n}^{2}}{\sigma^{2}}} .
$$

In our computations we fix lSNR and look at the corresponding tSNR value (and not the opposite). In such a way the error term is independent of the magnitude of $g^{0}$. Furthermore adding a nuisance function $g^{0}$ to model (8) increases at the same time the $t S N R$ and the difficulty of the estimation of the parameters. This is somehow not "fair". The classical signal to noise ratio for the standard Lasso (in our case the Lasso with known $g^{0}$ ) is lSNR. Fixing ISNR allows us to compare our results with other papers, like Bühlmann and Mandozzi (2013).

Table 1 resumes the initial conditions. 


\begin{tabular}{|l|c|}
\hline Setting parameter & \\
\hline \hline number $p$ of variables & 250 or 1000 \\
\hline sparsity $s_{0}$ & 5 or 20 \\
\hline linear signal to noise ratio (lSNR) & 2,8 or 32 \\
\hline function $g^{0}$ & $g_{1}^{0}, g_{2}^{0}$ or $g_{3}^{0}$ \\
\hline
\end{tabular}

Table 1: The parameter settings.

Other settings: A common choice for $J$ is $\int\left|g(z)^{\prime \prime}\right|^{2} d z$. For computational reasons we take $J^{2}(g):=\int\left|g(z)^{\prime \prime}\right|^{2} d z+c \int g^{2} d z$, where $c$ is small $\left(\approx 10^{-3}\right)$. Note that $J$ is a norm. We choose $\mu:=n^{-2 / 5} / 100$.

\subsection{Results}

Our aim is to compare our estimator with a well known estimator such the Lasso. In order to acquire a more global knowledge on the performance of our method different settings are chosen. In some of them the LK method performs very well (generally when $s_{0}$ is small and the lSNR is large) and in some others it is quite bad.

In Tables $2 / 5$ we summarize the results of the simulations. The performances of LK (see (10), LN (see (9)) and DP (11) are compared. The two different cases, where $X$ and $Z$ are independent and dependent are denoted with (DPi) and (DPd) respectively. Analogously we can define $L K i$ and $L K d$.

If $g^{0}$ is known, the dependence between $X$ and $Z$ does not play (given $X$ ) any role. Consequently LK scores very similarly in the dependent and in the independent case. In order to make our table more readable we just take the average of the score of LKi and LKd and denote it with LK. The same consideration holds for LN.

More in detail we compare the prediction error $\left\|\hat{Y}-Y_{0}\right\|_{n}$, the estimation error $\left\|\hat{\beta}-\beta^{0}\right\|_{1}$ and the true and false positive rate for $\hat{\beta}$ (TPR and FPR respectively). The error in the estimation of $g,\left\|\hat{g}-g^{0}\right\|_{2}$ is also given.

Hereafter we summarize the findings of our simulations.

\section{Prediction:}

As one can expect LK performs better than the other methods. This is not surprising because the nuisance model is more complex than the high-dimensional linear model. But this does not mean that our estimator is bad. In fact it works only slightly worse than LK in prediction terms. Compared to LN, DPi and 
DPd works in any design (for non-zero nuisance function) remarkably better. Finally we can remark that DP provides slightly better results if $X$ and $Z$ are independent, i.e. DPi has lower error than DPd. (See Figure 2).

\section{Estimation:}

First of all note that DP is the only method estimating both $\beta^{0}$ and $g^{0}$.

As theoretically shown in Theorem $3.2 \mathrm{LK}$ and DPi and DPd provide similar estimation for $\beta^{0}$. Similarly as for the prediction LK performs slightly better than DPi and DPd. DPi and DPd are remarkably better than LN, when LK (and consequently DP) works well. When all four methods works bad, then LN is only slightly worse than our methods. Compare Figure 3 and Tables 2 and

4 for quantitative results. The prediction of $\hat{\beta}$ is usually slightly worse in DPd than in DPi. This is not in contrast with Theorem 3.2, because in DPd the eigenvalue $\Lambda_{\tilde{X} \text {, min }}$ is smaller than in DPi.

\section{Estimation of the nuisance function:}

We now want to give a closer look at the estimation of the function $g^{0}$. From the results of the simulation we can conclude that

- The quality of the estimation of the function $g^{0}$ depends on the performance of $\hat{\beta}$. In general better estimation of $\beta^{0}$ means a better estimation of $g^{0}$.

- If an increase of ISNR improves the quality of the estimation of $\beta^{0}$, then it also improves the estimation of $g^{0}$ (see Figure 4 ).

- The function choice of $g^{0}$ does not play crucial role for the quality of the fit.

- Even if the estimation of $\beta^{0}$ (and consequently of $g^{0}$ ) are very bad, we can at least have an idea of "how the original function $g^{0}$ " looks like (see Figure 5).

Figure 4 shows the estimation of $g_{3}^{0}$ where $p=250, s_{0}=5$ and the signal to noise ratio varies between 2 and 32. As one can expect, improving SNR makes the estimation of $g^{0}$ more accurate.

Remark 4.3 (Estimation quality of $g^{0}$ for bad working LK method:). If LK does not properly estimate $\beta^{0}$, then we can not expect that our method does better. 

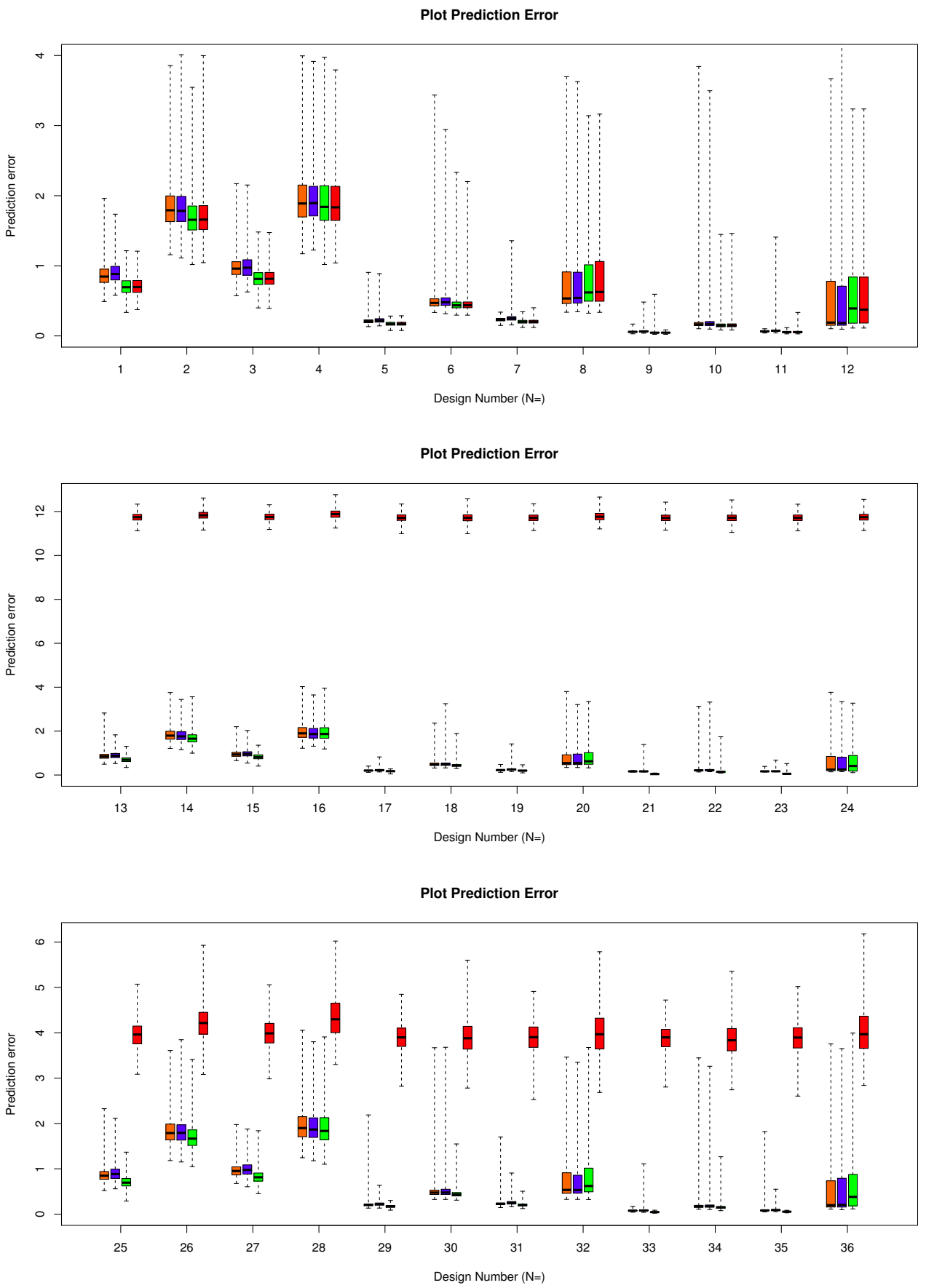

Figure 2: Prediction error for designs $N=1, \ldots, 36$ with dataset Leukemia. For each of the 36 designs the boxplots represent the prediction error for DPi (orange), DPd (blue) LK (green) and LN (red) based on 1000 replicates. 

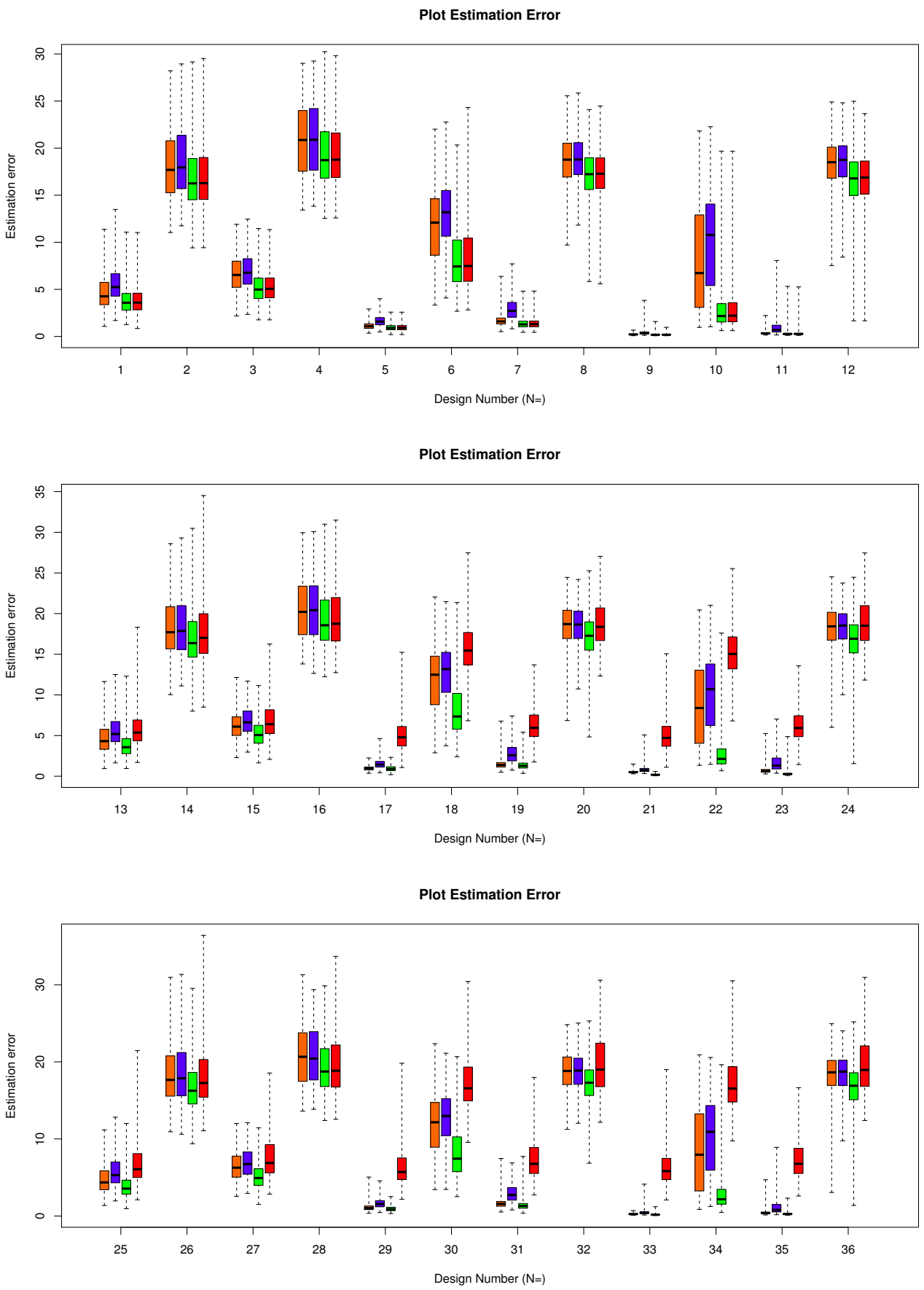

Figure 3: Prediction error for designs $N=1, \ldots, 36$ with dataset Leukemia. For each of the 36 designs the boxplots represent the estimation error for DPi (orange), DPd (blue) LK (green) and LN (red) based on 1000 replicates. 


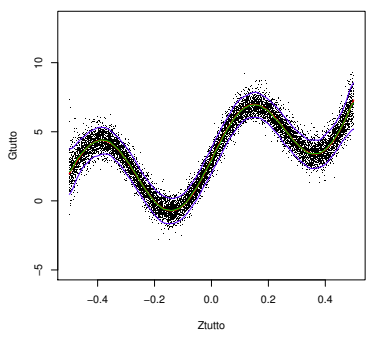

1a)

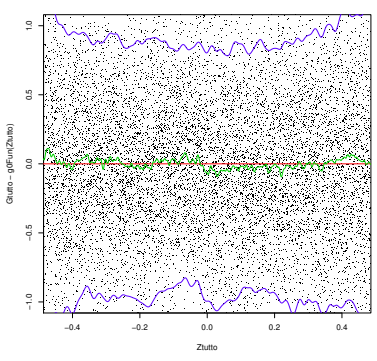

1b)

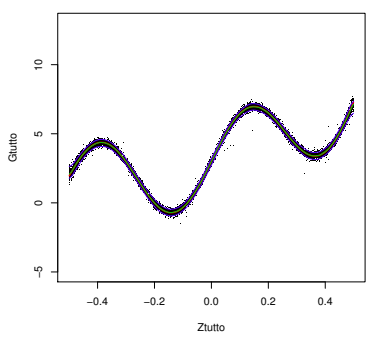

2a)

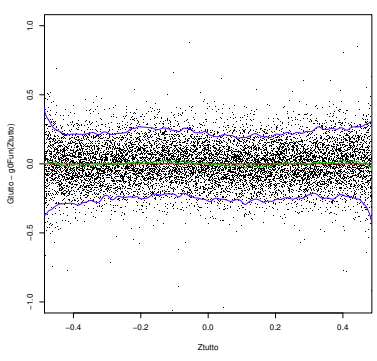

$2 b)$

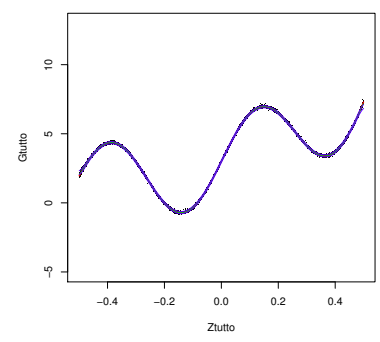

3a)

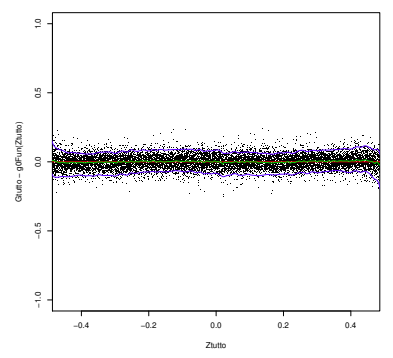

$3 \mathrm{~b})$

Figure 4: Estimation of the function $g^{0}$ for the designs $N=25,29,33$ respectively in dataset Leukemia. Here $p=250, s_{0}=5$ and the nuisance function $g_{3}$ are kept constant, whether $l S N R$ varies between 2 and 32. In the first row the estimated function is plotted, in the second the residuals. The red line represents the true function. The blue line corresponds to the mean of the estimated functions, and the blue lines define pointwise a $90 \%$ confidence interval. An increase of ISNR generally improves the estimation of $g^{0}$. 


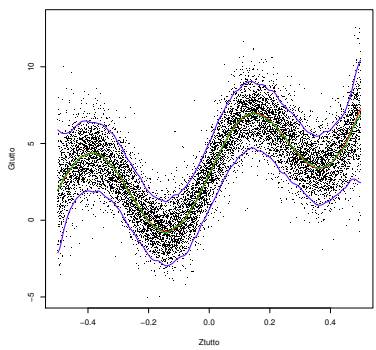

i)

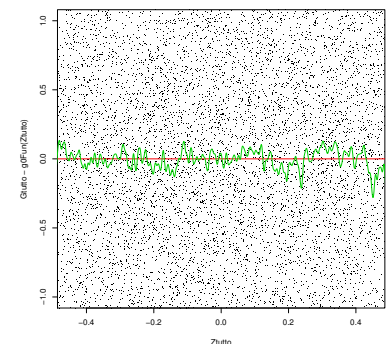

ii)

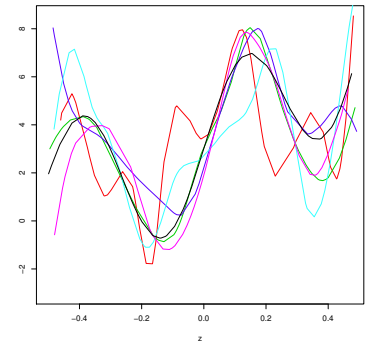

iii)

Figure 5: Estimation of the function $g^{0}$ for design $N=28\left(p=1000, s_{0}=\right.$ $15, S N R=2$ ). The first plot shows the true function $g^{0}$ (red), the mean of the estimated function on 1000 replicates (green) and $90 \%$ confidence bounds (blue). The coordinates of the black dots are $\left(z_{i}, g_{i}\right)$. In the second figure the residuals are plotted.

The third plot represents the function $g^{0}$ (black) and the estimated functions $\hat{g}$ for five (randomly chosen) replicates.

The estimation of $g^{0}$ is then also problematic, but even in this really bad and difficult case, it is possible to at least recognise some trends in $g^{0}$ and have an idea of the order of magnitude of $g^{0}$. Even if not totally satisfying this gives us a basic idea of how $g^{0}$ looks like.

We present as a prime example design number 28 (see Figure 5 , where $p=$ $1000, s_{0}=15$ and $S N R=2$. The plot shows that the $10 \%$ point wise confidence bounds more or less correspond to $g^{0} \pm 2.5$.

Looking at some randomly chosen results for the estimation of $g^{0}$, we are still able to somehow have an idea on how $g^{0}$ looks like, even if the estimation is very rude.

\section{Limit cases:}

Here we briefly discuss, without presenting all numerical results, some extreme cases.

Very large $g^{0}$ : If the nuisance function completely dominates the linear part the estimation of $g^{0}$ is easier (small relative error) than in the previous cases, but we pay in term of estimation of $\beta^{0}$. For example for $g^{0}(z):=200 g_{3}^{0}(z)+400$, even if LK has a TPR of $100 \%$, our method is on average not able to pick out more than $28-32 \%$ of the true positives. This is in any case better than LN, which in this case scores similarly as just randomly selecting components.

The tuning parameter $\mu$ In our design $\mu$ is chosen as $\mu_{0}:=n^{-2 / 5} / 100$. The 
choice of $\mu$ seems to play a marginal role in the estimation quality. We tried to repeat the simulations with some different values of $\mu$. Even multiplying or dividing $\mu_{0}$ by a factor 10 does not substantially affect our results.

Our estimator is then quite robust in $\mu$, but as one can expect extreme values of $\mu$ (like $\mu=10000 \cdot \mu_{0}$ ) leads to problematic estimation of $g^{0}$.

Very highly correlated covariates If $Z$ is strongly correlated with a linear combination of the active part of $X$, then $\Lambda_{\tilde{X} \text {, min }}$ is (almost) equal to zero. This entails that the estimation is bad, but we can anyway still have appreciable prediction results.

\section{Conclusions}

As partial linear models have been successfully applied, and the parametric part can be estimated with parametric rate, it is both from practical as well as theoretical point of view of interest to extend the model and theory to the case where the linear part is high-dimensional. As we have shown, the linear part can be estimated with oracle rates as if the nuisance function were known, even though the rate for estimating the nuisance function is slower than the oracle rate for the linear part.

Our method of proof makes use of empirical process theory, in particular it shows uniform probability inequalities for empirical $L_{2}$-norms. It moreover shows bounds for empirical correlations between transformed observations, uniformly over a class of transformations.

The simulations show that it indeed may pay off to include the nuisance function into the model. From computational point of view, the methodology does not impose additional difficulties when using quadratic penalties for the nuisance part.

\section{Proofs}

\subsection{Sketch of proofs}

We prove Theorem 3.1 in two steps. We first assume that we are under some event $\mathcal{T}$. Conditionally on $\mathcal{T}$ all conclusions of Theorem 3.1 hold with probability 1 (see Lemma 6.1). Then we show that the event $\mathcal{T}$ has large probability, 
at least $1-3 \exp \left[-n \delta_{T}^{2} \mu^{2}\right]$ (see Lemmas 6.2 6.6). This ends the proof. Theorem 3.2 supplies stronger results. The proof goes along the lines of the first theorem but uses the first theorem as starting point.

\subsection{The result assuming empirical process conditions}

Let for each $R>0$

$$
\mathcal{F}(R):=\left\{f: \tau_{\mu, R}(f) \leq R\right\}
$$

and

$$
\mathcal{T}\left(\delta_{0}, R\right):=\mathcal{T}_{1}\left(\delta_{0}, R\right) \cap \mathcal{T}_{2}\left(\delta_{0}, R\right),
$$

where

$$
\mathcal{T}_{1}\left(\delta_{0}, R\right):=\left\{(X, Z): \sup _{f \in \mathcal{F}(R)}\left|\|f\|_{n}^{2}-\|f\|^{2}\right| \leq \delta_{0} R^{2}\right\}
$$

and

$$
\mathcal{T}_{2}\left(\delta_{0}, R\right):=\left\{(X, Z, E):\left|\sup _{f \in \mathcal{F}(R)}\right| E^{T} f / n \mid \leq \delta_{0} R^{2}\right\} .
$$

Lemma 6.1. Let $\mu^{2} \leq \delta_{0} R^{2} /\left(2 J^{2}\left(g^{0}\right)\right)$. Assume Condition 2.3 and that

$$
\frac{4 \lambda^{2} s_{0}}{\Lambda_{\tilde{X}, \min }^{2}} \leq \delta_{0} R^{2}
$$

Then on $\mathcal{T}\left(\delta_{0}, R\right)$, it holds that $\tau\left(\hat{f}-f^{0}\right) \leq R$.

Proof. Define

$$
\tilde{f}:=s \hat{f}+(1-s) f^{0}=X \tilde{\beta}+\tilde{g},
$$

where $\tilde{\beta}=s \hat{\beta}+(1-s) \beta^{0}$ and $\tilde{g}=s \hat{g}+(1-s) g^{0}$ and

$$
s:=\frac{R}{R+\tau\left(\hat{f}-f^{0}\right)} .
$$

We prove this lemma with the following trick. The function $\tilde{f}$ is defined in such a way that $\tau\left(\tilde{f}-f^{0}\right) \leq R$. Then on $\mathcal{T}$, from the "basic inequality" below, we get $\tau\left(\tilde{f}-f^{0}\right) \leq R / 2$. This implies $\tau\left(\hat{f}-f^{0}\right) \leq R$.

We now present the details of this trick. By convexity and definition of $\hat{f}$, we know that

$$
\begin{gathered}
\|Y-\tilde{f}\|_{n}^{2}+\lambda\|\tilde{\beta}\|_{1}+\mu^{2} J^{2}(\tilde{g}) \leq \\
s\left(\|Y-\hat{f}\|_{n}^{2}+\lambda\|\hat{\beta}\|_{1}+\mu^{2} J^{2}(\hat{g})\right)+(1-s)\left(\left\|Y-f^{0}\right\|_{n}^{2}+\lambda\left\|\beta^{0}\right\|_{1}+\mu^{2} J^{2}\left(g^{0}\right)\right)
\end{gathered}
$$




$$
\leq\left\|Y-f^{0}\right\|_{n}^{2}+\lambda\left\|\beta^{0}\right\|_{1}+\mu^{2} J^{2}\left(g^{0}\right) .
$$

This is the "basic inequality". Using that $Y=f^{0}+E$ rewrite this to

$$
\left\|\tilde{f}-f^{0}\right\|_{n}^{2}+\lambda\|\tilde{\beta}\|_{1}+\mu^{2} J^{2}(\tilde{g}) \leq 2 E^{T}\left(\tilde{f}-f^{0}\right) / n+\lambda\left\|\beta^{0}\right\|_{1}+\mu^{2} J^{2}\left(g^{0}\right) .
$$

Now use that

$$
\tau\left(\tilde{f}-f^{0}\right)=s \tau\left(\hat{f}-f^{0}\right)=\frac{R \tau\left(\hat{f}-f^{0}\right)}{R+\tau\left(\hat{f}-f^{0}\right)} \leq R .
$$

Hence on $\mathcal{T}\left(\delta_{0}, R\right)$, we find

$$
\left\|\tilde{f}-f^{0}\right\|^{2}+\lambda\|\tilde{\beta}\|_{1}+\mu^{2} J^{2}(\tilde{g}) \leq 3 \delta_{0} R^{2}+\lambda\left\|\beta^{0}\right\|_{1}+\mu^{2} J^{2}\left(g^{0}\right) .
$$

Using Equation (3), the identity $\|\tilde{\beta}\|_{1}=\left\|\tilde{\beta}_{S_{0}^{c}}\right\|_{1}+\left\|\tilde{\beta}_{S_{0}}\right\|_{1}$ and then the triangle inequality, we obtain

$$
\begin{gathered}
\left\|\tilde{X}\left(\tilde{\beta}-\beta^{0}\right)\right\|^{2}+\left\|h\left(\tilde{\beta}-\beta^{0}\right)+\left(\tilde{g}-g^{0}\right)\right\|^{2}+\lambda\left\|\tilde{\beta}_{S_{0}^{c}}\right\|_{1}+\mu^{2} J^{2}(\tilde{g}) \\
\leq 3 \delta_{0} R^{2}+\mu^{2} J^{2}\left(g^{0}\right)+\lambda\left\|\beta^{0}-\tilde{\beta}_{S_{0}}\right\|_{1} .
\end{gathered}
$$

Using the approximation $u v \leq u^{2}+\frac{v^{2}}{4}$ and the assumptions of the lemma, we get

$$
\begin{aligned}
\lambda\left\|\tilde{\beta}_{S_{0}}-\beta^{0}\right\|_{1} & \leq \lambda \sqrt{s_{0}}\left\|\tilde{\beta}_{S_{0}}-\beta^{0}\right\| \leq \lambda \sqrt{s_{0}}\left\|\tilde{\beta}-\beta^{0}\right\| \\
& \leq \frac{\lambda \sqrt{s_{0}}}{\Lambda_{\tilde{X}, \min }}\left\|\tilde{X}\left(\tilde{\beta}-\beta_{0}\right)\right\| \leq \frac{\lambda^{2} s_{0}}{\Lambda_{\tilde{X}, \min }^{2}}+\frac{\left\|\tilde{X}\left(\tilde{\beta}-\beta_{0}\right)\right\|^{2}}{4} \\
& \leq \frac{\delta_{0} R^{2}}{4}+\frac{\left\|\tilde{X}\left(\tilde{\beta}-\beta_{0}\right)\right\|^{2}}{4}
\end{aligned}
$$

and similarly

$$
2 \lambda\left\|\tilde{\beta}_{S_{0}}-\beta^{0}\right\|_{1} \leq \frac{\lambda^{2} s_{0}}{\Lambda_{\tilde{X}, \text { min }}^{2}}+\left\|\tilde{X}\left(\tilde{\beta}-\beta_{0}\right)\right\|^{2} \leq \frac{\delta_{0} R^{2}}{4}+\left\|\tilde{X}\left(\tilde{\beta}-\beta_{0}\right)\right\|^{2} .
$$

Note now that using $4 u v \leq 4 u^{2}+v^{2}$ it yields

$$
\begin{gathered}
\frac{3}{4} J^{2}\left(\tilde{g}-g^{0}\right)=\frac{3}{4}\left(J^{2}(\tilde{g})+J^{2}\left(g^{0}\right)\right)+\frac{3}{2} J\left(\tilde{g}, g^{0}\right) \\
\leq \frac{3}{4}\left(J^{2}(\tilde{g})+J^{2}\left(g^{0}\right)\right)+\frac{1}{4} \cdot 4 \cdot \frac{3}{2} J\left(\tilde{g}, g^{0}\right)
\end{gathered}
$$




$$
\leq \frac{3}{4}\left(J^{2}(\tilde{g})+J^{2}\left(g^{0}\right)\right)+\frac{9}{4} J^{2}\left(g^{0}\right)+\frac{1}{4} J^{2}(\tilde{g})=J^{2}(\tilde{g})+3 J^{2}\left(g^{0}\right)
$$

Adding on both sides of inequality (12) $\frac{3}{4} \mu^{2} J^{2}\left(\tilde{g}-g^{0}\right)$ and using successively Inequalities (13) and (15) this

leads to

$$
\begin{aligned}
& \left\|\tilde{X}\left(\tilde{\beta}-\beta^{0}\right)\right\|^{2}+\left\|h\left(\tilde{\beta}-\beta^{0}\right)+\left(\tilde{g}-g^{0}\right)\right\|^{2}+\frac{3}{4} \mu^{2} J^{2}\left(\tilde{g}-g^{0}\right) \\
\leq & 3 \delta_{0} R^{2}+4 \mu^{2} J^{2}\left(g^{0}\right)+\frac{\delta_{0} R^{2}}{4}+\frac{1}{4}\left\|\tilde{X}\left(\tilde{\beta}-\beta^{0}\right)\right\|^{2}-\lambda\left\|\tilde{\beta}_{S_{0}^{c}}\right\|_{1} .
\end{aligned}
$$

By the assumption $\mu^{2} \leq \delta_{0} R^{2} /\left(2 J^{2}\left(g^{0}\right)\right)$ we obtain

$$
\begin{gathered}
\frac{3}{4}\left\|\tilde{X}\left(\tilde{\beta}-\beta^{0}\right)\right\|^{2} \\
+\left\|h\left(\tilde{\beta}-\beta^{0}\right)+\left(\tilde{g}-g^{0}\right)\right\|^{2}+\frac{3}{4} \mu^{2} J^{2}\left(\tilde{g}-g^{0}\right) \\
\leq 3 \delta_{0} R^{2}+2 \delta_{0} R^{2}+\frac{1}{4} \delta_{0} R^{2} .
\end{gathered}
$$

Hence, by Remark 3.1

$$
\left\|\tilde{f}-f^{0}\right\|^{2}+\mu^{2} J^{2}\left(\tilde{g}-g^{0}\right) \leq 7 \delta_{0} R^{2} .
$$

Therefore

$$
\left(\left\|X\left(\tilde{\beta}-\beta^{0}\right)+\left(\tilde{g}-g^{0}\right)\right\|^{2}+\mu^{2} J^{2}(\tilde{g})\right)^{1 / 2} \leq\left(7 \delta_{0}\right)^{1 / 2} R
$$

On the other hand, adding now $\lambda\left\|\tilde{\beta}_{S_{0}}-\beta^{0}\right\|_{1}$ on both sides of 12 leads to

$$
\begin{gathered}
\left\|\tilde{X}\left(\tilde{\beta}-\beta^{0}\right)\right\|^{2}+\left\|h\left(\tilde{\beta}-\beta^{0}\right)+\left(\tilde{g}-g^{0}\right)\right\|^{2}+\lambda\left\|\tilde{\beta}-\beta^{0}\right\|_{1}+\mu^{2} J^{2}(\tilde{g}) \\
\leq 3 \delta_{0} R^{2}+\mu^{2} J^{2}\left(g^{0}\right)+2 \lambda\left\|\beta^{0}-\tilde{\beta}_{S_{0}}\right\|_{1} \\
\leq \frac{15}{4} \delta_{0} R^{2}+\left\|\tilde{X}\left(\tilde{\beta}-\beta^{0}\right)\right\|^{2}
\end{gathered}
$$

where in the last inequality the assumption $\mu^{2} \leq \delta_{0} R^{2} /\left(2 J^{2}\left(g^{0}\right)\right)$ and Inequality (14) were applied.

We now have:

$$
\lambda\left\|\tilde{\beta}-\beta^{0}\right\|_{1} \leq \frac{15}{4} \delta_{0} R^{2}
$$

and

$$
\frac{\lambda\left\|\tilde{\beta}-\beta^{0}\right\|_{1}}{R \sqrt{\delta_{0} / 2}} \leq \frac{15}{4} \sqrt{2 \delta_{0}} R .
$$


Then

$$
\tau\left(\tilde{f}-f^{0}\right) \leq\left(7 \delta_{0}\right)^{1 / 2} R+\frac{15}{4} \sqrt{2 \delta_{0}} R \leq \frac{\sqrt{253 \delta_{0}}}{2} R=\frac{R}{2},
$$

Thus we have

$$
\tau\left(\tilde{f}-f^{0}\right)=s \tau\left(\hat{f}-f^{0}\right)=\frac{R \tau\left(\hat{f}-f^{0}\right)}{R+\tau\left(\hat{f}-f^{0}\right)} \leq \frac{R}{2} .
$$

Hence

$$
\tau\left(\hat{f}-f^{0}\right) \leq R
$$

\section{Technical tools}

The main goal of the following lemmas is to prove that the set $\mathcal{T}$ has large probability.

The next lemma is along the lines of Theorem 3.1 in Giné and Koltchinskii (2006).

Lemma 6.2. Let $\mathcal{G}(\tilde{R}, \tilde{K})$ be a class of functions $g$ on $\mathbb{R}^{d}$, uniformly bounded by $\tilde{K}$, and satisfying $\|g\| \leq \tilde{R}$. Let $\left\{\varepsilon_{i}\right\}_{i=1}^{n}$ be a Rademacher sequence independent of $\left\{z_{i}\right\}_{i=1}^{n}$, that is $\varepsilon_{i}$ are i.i.d. and take values \pm 1 with probability $1 / 2$ each. Suppose that for some increasing strictly convex function $G$, it holds that

$$
\mathbb{E} \sup _{g \in \mathcal{G}(\tilde{R}, \tilde{K})}\left|\frac{1}{n} \sum_{i=1}^{n} g_{i} \varepsilon_{i}\right| \leq \mathbb{E} G^{-1}\left(\tilde{R}_{n}^{2}\right) / \sqrt{n}
$$

where

$$
\tilde{R}_{n}^{2}=\sup _{g \in \mathcal{G}(\tilde{R}, \tilde{K})}\|g\|_{n}^{2}
$$

Let $H$ be the convex conjugate of $G$. Then for $\tilde{R}^{2} \geq H(16 \tilde{K} / \sqrt{n}) / 2$, we have

$$
\mathbb{E} G^{-1}\left(\tilde{R}_{n}^{2}\right) \leq G^{-1}\left(4 \tilde{R}^{2}\right)
$$

and

$$
\mathbb{E} \sup _{g \in \mathcal{G}(\tilde{R}, \tilde{K})}\left|\|g\|_{n}^{2}-\|g\|^{2}\right| \leq 8 \tilde{K} G^{-1}\left(4 \tilde{R}^{2}\right) / \sqrt{n}
$$

Proof. By symmetrization we have (see e.g. van der Vaart and Wellner (1996)),

$$
\mathbb{E} \sup _{g \in \mathcal{G}(\tilde{R}, \tilde{K})}\left|\|g\|_{n}^{2}-\|g\|^{2}\right|=\mathbb{E} \sup _{g \in \mathcal{G}(\tilde{R}, \tilde{K})}\left|\frac{1}{n} \sum_{i=1}^{n}\left(g_{i}^{2}-\mathbb{E} g_{i}^{2}\right)\right|
$$




$$
\begin{aligned}
& \leq 2 \mathbb{E} \sup _{g \in \mathcal{G}(\tilde{R}, \tilde{K})}\left|\frac{1}{n} \sum_{i=1}^{n} g_{i}^{2} \varepsilon_{i}\right| \\
& \leq 8 \tilde{K} \mathbb{E} \sup _{g \in \mathcal{G}(\tilde{R}, \tilde{K})}\left|\frac{1}{n} \sum_{i=1}^{n} g_{i} \varepsilon_{i}\right|,
\end{aligned}
$$

where in the last inequality we used the contraction inequality of Ledoux and Talagrand (1991) for Lipschitz loss function (see Theorem 14.4 of Bühlmann and van de Geer (2011)). But by assumption:

$$
\mathbb{E} \sup _{g \in \mathcal{G}(\tilde{R}, \tilde{K})}\left|\frac{1}{n} \sum_{i=1}^{n} g_{i} \varepsilon_{i}\right| \leq \mathbb{E} G^{-1}\left(\tilde{R}_{n}^{2}\right) / \sqrt{n} .
$$

Thus we have for $\tilde{u}:=\mathbb{E} G^{-1}\left(\tilde{R}_{n}^{2}\right)$,

$$
\mathbb{E} \tilde{R}_{n}^{2} \leq \sup _{g \in \mathcal{G}(\tilde{R}, \tilde{K})}\|g\|^{2}+\mathbb{E} \sup _{g \in \mathcal{G}(\tilde{R}, \tilde{K})}\left|\|g\|_{n}^{2}-\|g\|^{2}\right| \leq \tilde{R}^{2}+8 \tilde{K} \tilde{u} / \sqrt{n}
$$

On the other hand, by Jensen's inequality,

$$
\mathbb{E} \tilde{R}_{n}^{2}=\mathbb{E} G\left(G^{-1}\left(\tilde{R}_{n}^{2}\right)\right) \geq G\left(\mathbb{E} G^{-1}\left(\tilde{R}_{n}^{2}\right)\right)=G(\tilde{u}) .
$$

Let

$$
H(v):=\sup _{u}\{v u-G(u)\}
$$

be the convex conjugate of $G$, then we have

$$
G(\tilde{u}) \leq \tilde{R}^{2}+8 \tilde{K} \tilde{u} / \sqrt{n} \leq \tilde{R}^{2}+G(\tilde{u}) / 2+H(16 \tilde{K} / \sqrt{n}) / 2 .
$$

and by the assumption

$$
G(\tilde{u}) \leq 2 \tilde{R}^{2}+H(16 \tilde{K} / \sqrt{n}) \leq 4 \tilde{R}^{2} .
$$

In other words, we showed that

$$
G\left(\mathbb{E} G^{-1}\left(\tilde{R}_{n}^{2}\right)\right) \leq 4 \tilde{R}^{2}
$$

$G$ is monotone, so

$$
\mathbb{E} G^{-1}\left(\tilde{R}_{n}^{2}\right) \leq G^{-1}\left(4 \tilde{R}^{2}\right)
$$


Lemma 6.3. Assume the conditions of Lemma 6.2. Then, for all $\tilde{R}^{2} \geq$ $H(16 \tilde{K} / \sqrt{n}) / 2$,

$$
\mathbb{E} \sup _{g \in(\tilde{R}, \tilde{K})}\left|\frac{1}{n} \sum_{i=1}^{n} g_{i} \epsilon_{i}\right| \leq G^{-1}\left(4 \tilde{R}^{2}\right) / \sqrt{n}
$$

and for all $\delta>0$,

$$
\mathbb{E} \sup _{g \in \mathcal{G}(\tilde{R}, \tilde{K})}\left|\|g\|_{n}^{2}-\|g\|^{2}\right| \leq 4 \delta \tilde{R}^{2}+\delta H\left(\frac{8 \tilde{K}}{\delta \sqrt{n}}\right) .
$$

Proof. Along the lines of Lemma 6.2, starting from 16 we get

$$
\mathbb{E} \sup _{g \in \mathcal{G}(\tilde{R}, \tilde{K})}\left|\|g\|_{n}^{2}-\|g\|^{2}\right| \leq 8 \tilde{K} \tilde{u} / \sqrt{n}
$$

The right hand side of the above inequality can be further approximate by

$$
8 \tilde{K} \tilde{u} / \sqrt{n} \leq \delta G(\tilde{u})+\sup _{u}\left\{\frac{8 \tilde{K} u}{\sqrt{n}}-\delta G(u)\right\} \leq 4 \delta \tilde{R}^{2}+\delta H\left(\frac{8 \tilde{K}}{\delta \sqrt{n}}\right) .
$$

\subsection{The set $\mathcal{T}_{1}\left(\delta_{0}, R\right)$}

Lemma 6.4. Let for a fixed $\beta$

$$
R_{n}^{2}:=\sup _{g: X \beta+g \in \mathcal{F}(R)}\|g\|_{n}^{2}
$$

Assume Conditions 2.3 and 2.4, that $\mu \leq R$ and (7), then

$$
\mathbb{E} \sup _{g: X \beta+g \in \mathcal{F}(R)}\left|\frac{1}{n} \sum_{i=1}^{n} g_{i} \varepsilon_{i}\right| \leq \mathbb{E} G^{-1}\left(R_{n}^{2}\right) / \sqrt{n},
$$

where $\varepsilon_{1}, \ldots, \varepsilon_{n}$ is a Rademacher sequence independent of $\left\{z_{i}\right\}_{i=1}^{n}$, and where

$$
G(u):=\kappa_{0}^{\prime}(R / \mu)^{-\frac{2}{2 m-1}} u^{\frac{4 m}{2 m-1}}, u>0,
$$

with $\kappa_{0}^{\prime}$ a constant depending only on $A$ and $m$. Moreover, if in addition Condition 2.5 holds, we have for a constant $\kappa_{0}$ depending only on $A, m, \Lambda$ (defined in (7)) and $K_{g}$, and for $\mu^{2} \geq \kappa_{0} n^{-\frac{2 m}{2 m+1}}$, 


$$
\mathbb{E} \sup _{g: X \beta+g \in \mathcal{F}(R)}\left|\frac{1}{n} \sum_{i=1}^{n} g_{i} \varepsilon_{i}\right| \leq \kappa_{0} \frac{R^{1-\frac{1}{2 m}}}{\sqrt{n}}[R / \mu]^{\frac{1}{2 m}}
$$

and for $\mu^{2} \geq\left(\kappa_{0} \vee \delta_{1}^{-\frac{4 m}{2 m+1}}\right) n^{-\frac{2 m}{2 m+1}}$

$$
\mathbb{E} \sup _{g: X \beta+g \in \mathcal{F}(R)}\left|\|g\|_{n}^{2}-\|g\|^{2}\right| \leq 8 K_{g} \kappa_{0} \delta_{1} R^{2} .
$$

Suitable choices for $\delta_{1}, \kappa_{0}$ and $\kappa_{0}^{\prime}$ are

$$
\begin{gathered}
\delta_{1} \leq\left(8 C \sqrt{A} K_{g}\right)^{-1}\left[\frac{2 m+1}{2 \Lambda^{2}(2 m-1)}\right]^{-\frac{2 m+1}{4 m}} \\
\kappa_{0}:=C \sqrt{A} \frac{2 m}{2 m-1}(2 \Lambda)^{\frac{2 m-1}{2 m+1}} \\
\kappa_{0}^{\prime}:=\left(C \sqrt{A} \frac{2 m}{2 m-1}\right)^{-\frac{4 m}{2 m-1}}
\end{gathered}
$$

and results from (25), 26) and (22) respectively. Here, $C$ is an appropriate universal constant (coming from Dudley's inequality).

Note that the proposed function $G$ is strictly convex, positive and increasing as required in Lemma 6.2 and that the assumption $\mu \leq R$ is less restrictive than Assumption (4), as required in Theorem 3.1.

Proof. If $X \beta+g \in \mathcal{F}(R)$, we have by Remark 3.1 $\|h \beta+g\|^{2} \leq R^{2}$ as well as $\|\tilde{X} \beta\|^{2} \leq R^{2}$. Hence, $\|\beta\|_{2}^{2} \leq R^{2} / \Lambda_{\tilde{X} \text {, min }}^{2}$, so that $\|h \beta\|^{2} \leq R^{2} \Lambda_{h, \max }^{2} / \Lambda_{\tilde{X}, \min }^{2}$. Thus

$$
\|g\| \leq\|h \beta+g\|+\|h \beta\| \leq\left(1+\frac{\Lambda_{h, \max }}{\Lambda_{\tilde{X}, \min }}\right) R:=\Lambda R .
$$

and

$$
\left\|\frac{\mu g}{R}\right\|^{2} \leq \mu^{2} \Lambda^{2} \leq 1
$$

Furthermore, $\tau(f) \leq R$ implies

$$
J^{2}(g) \leq R^{2} / \mu^{2}
$$

Hence

$$
J\left(\frac{\mu g}{R}\right) \leq 1
$$


By Condition 2.4 we have

$$
\begin{aligned}
& \mathcal{H}\left(u,\{g: X \beta+g \in \mathcal{F}(R)\},\|\cdot\|_{\infty}\right) \\
\leq & \mathcal{H}\left(u,\left\{g: J^{2}(g \mu / R) \leq 1 ;\|g \mu / R\|^{2} \leq 1\right\},\|\cdot\|_{\infty}\right) \\
= & \mathcal{H}\left(\frac{u \mu}{R}, g: J^{2}(g) \leq 1 ;\|g\|^{2} \leq 1,\|\cdot\|_{\infty}\right) \\
\leq & A\left(\frac{R}{u \mu}\right)^{\frac{1}{m}}, \quad u>0 .
\end{aligned}
$$

By Dudley's inequality (see e.g. van der Vaart and Wellner (1996)) we get for a universal constant $C$

$$
\begin{aligned}
& \mathbb{E} \sup _{g: X \beta+g \in \mathcal{F}(R)}\left|\frac{1}{n} \sum_{i=1}^{n} g_{i} \varepsilon_{i}\right| \\
\leq & \mathbb{E} \frac{C}{\sqrt{n}} \int_{0}^{R_{n}}\left(\mathcal{H}\left(u,\{g: X \beta+g \in \mathcal{F}(R)\},\|\cdot\|_{\infty}\right)\right)^{\frac{1}{2}} d u \\
\leq & \mathbb{E} \frac{C}{\sqrt{n}} \int_{0}^{R_{n}} \sqrt{A}\left(\frac{R}{u \mu}\right)^{\frac{1}{2 m}} d u \\
= & \mathbb{E} C \sqrt{A} \frac{2 m}{2 m-1}\left(\frac{R_{n}^{\frac{2 m-1}{2 m}}}{\sqrt{n}}\right)\left(\frac{R}{\mu}\right)^{\frac{1}{2 m}} \\
= & \mathbb{E} G^{-1}\left(R_{n}^{2}\right) / \sqrt{n}
\end{aligned}
$$

Where

$$
G^{-1}\left(R_{n}^{2}\right):=C \sqrt{A} \frac{2 m}{2 m-1} R_{n}^{\frac{2 m-1}{2 m}}[R / \mu]^{\frac{1}{2 m}} .
$$

One can now easily see that $G^{-1}$ is the inverse of $G$ defined in (18).

Let now $\mathcal{G}(\tilde{R}, \tilde{K}):=\left\{g: X \beta+g \in \mathcal{F}(R),\|g\| \leq \tilde{R},\|g\|_{\infty} \leq \tilde{K}\right\}$.

Note that by Condition 2.5, for $X \beta+g \in \mathcal{F}(R)$,

$$
\|g\|_{\infty} \leq K_{g} R / \mu
$$

Take now $\tilde{R}:=\Lambda R$ and $\tilde{K}:=K_{g} R / \mu$, then the conditions of Lemma 6.2 are fulfilled. After some easy computations we furthermore get

$$
H(v)=\kappa_{0}^{\prime \prime}(v)^{\frac{4 m}{2 m+1}}[R / \mu]^{\frac{2}{2 m+1}}
$$


where

$$
\kappa_{0}^{\prime \prime}:=\left[C \sqrt{A} \frac{2 m}{2 m-1}\right]^{\frac{4 m}{2 m+1}} \cdot \frac{(2 m+1)(2 m-1)^{\frac{2 m-1}{2 m+1}}}{(4 m)^{\frac{4 m}{2 m+1}}}
$$

and

$$
\begin{aligned}
H\left(\frac{16 \tilde{K}}{\sqrt{n}}\right) & =\left[8 C \sqrt{A} K_{g}\right]^{\frac{4 m}{2 m+1}} \frac{2 m+1}{2 m-1} n^{-\frac{2 m}{2 m-1}}[R / \mu]^{2} \\
\leq & {\left[8 C \sqrt{A} K_{g}\right]^{\frac{4 m}{2 m+1}} \frac{2 m+1}{2 m-1} \delta_{1}^{\frac{4 m}{2 m+1}} R^{2} } \\
\leq & 2 \Lambda^{2} R^{2}
\end{aligned}
$$

Consequently from Lemma 6.2 we have

$$
\begin{aligned}
\mathbb{E} G^{-1}\left(R_{n}^{2}\right) / \sqrt{n} & \leq G^{-1}\left(4 \Lambda^{2} R^{2}\right) / \sqrt{n} \\
& =C \sqrt{A} \frac{2 m}{2 m-1}(2 \Lambda)^{\frac{2 m-1}{2 m+1}} \frac{R^{1-\frac{1}{2 m}}}{\sqrt{n}}[R / \mu]^{\frac{1}{2 m}}
\end{aligned}
$$

Resuming we showed

$$
\begin{aligned}
\mathbb{E} \sup _{g: X \beta+g \in \mathcal{F}(R)}\left|\frac{1}{n} \sum_{i=1}^{n} g_{i} \varepsilon_{i}\right| & \leq C \sqrt{A} \frac{2 m}{2 m-1}(2 \Lambda)^{\frac{2 m-1}{2 m+1}} \frac{R^{1-\frac{1}{2 m}}}{\sqrt{n}}[R / \mu]^{\frac{1}{2 m}} \\
& \leq \kappa_{0} \frac{R^{1-\frac{1}{2 m}}}{\sqrt{n}}[R / \mu]^{\frac{1}{2 m}} .
\end{aligned}
$$

As in the proof of Lemma 6.2 and Lemma 6.3

$$
\mathbb{E} \sup _{g: X \beta+g \in \mathcal{F}(R)}\left|\|g\|_{n}^{2}-\|g\|^{2}\right| \leq 8 \tilde{K} \mathbb{E} G^{-1}\left(\tilde{R}_{n}^{2}\right) / \sqrt{n} .
$$

The right hand side of the inequality can be further approximated by

$$
\begin{aligned}
8 K \mathbb{E} G^{-1}\left(\tilde{R}_{n}^{2}\right) / \sqrt{n} & \leq \frac{8 K_{g} R}{\mu \sqrt{n}} \mathbb{E} G^{-1}\left(\tilde{R}_{n}^{2}\right) \\
& \leq \frac{8 K_{g} R}{\mu \sqrt{n}} \mathbb{E} G^{-1}\left(4 \Lambda R^{2}\right) \\
& \leq \frac{8 K_{g} R}{\mu} C \sqrt{A} \frac{2 m}{2 m-1}(2 \Lambda)^{\frac{2 m-1}{2 m+1}} \frac{R^{1-\frac{1}{2 m}}}{\sqrt{n}}[R / \mu]^{\frac{1}{2 m}} \\
& \leq 8 K_{g} \kappa_{0} R^{2} \mu^{-\frac{2 m+1}{2 m}} n^{-\frac{1}{2}} \\
& \leq 8 K_{g} \kappa_{0} \delta_{1} R^{2} .
\end{aligned}
$$


This ends the proof.

In the following lemma we finally show that the set $\mathcal{T}_{1}$ has large probability. First we present a remark that will be useful for the proof.

Remark 6.1. It holds that

$$
\begin{array}{r}
\mathbb{E} R_{n} \leq\left(\mathbb{E}\left(R_{n}^{2}\right)\right)^{\frac{1}{2}} \leq \sqrt{\sup _{g: X \beta+g \in \mathcal{F}(R)}\left|\|g\|_{n}^{2}-\|g\|^{2}\right|+\|g\|^{2}} \\
\leq \sqrt{8 K_{g} \kappa_{0} \delta_{1} R^{2}+\Lambda^{2} R^{2}}=R \sqrt{8 K_{g} \kappa_{0} \delta_{1}+\Lambda^{2}}
\end{array}
$$

with $\Lambda=1+\Lambda_{\tilde{X} \text {, } \min } / \Lambda_{h, \max }$. Here we used Jensen inequality for the first inequality and the proof of Lemma 6.4 for the second one step.

Lemma 6.5. Assume Conditions 2.2, 2.3, 2.4 and 2.5, that $\mu \leq R$, (7) and that $R^{2} \leq \lambda \leq 1$.

Then for constants $\delta_{1}^{\prime}$ and $\kappa_{1}^{\prime}$ depending only on $K_{X}, K_{g}, A, m, \Lambda$ (defined in (7)), for $\delta_{1}^{\prime} \lambda \geq \lambda_{0}$, with $\lambda_{0}=\sqrt{2 \log (2 p) / n}$, and

$$
\mu^{2} \geq \kappa_{1}^{\prime} n^{-\frac{2 m}{2 m+1}}
$$

we have

$$
\sup _{f \in \mathcal{F}(R)}\left|\|f\|_{n}^{2}-\|f\|^{2}\right| \leq \delta_{0} R^{2}
$$

with probability at least $1-\exp \left[-n\left(\delta_{1}^{\prime}\right)^{2} \mu^{2}\right]$.

A suitable choice for $\kappa_{1}^{\prime}$ is

$$
\kappa_{1}^{\prime}:=\frac{C \sqrt{8 K_{g} \kappa_{0} \delta_{1}+\Lambda^{2}}}{\sqrt{2}}+\kappa_{0} K_{X}^{\frac{1}{2 m}} \sqrt{\delta_{0} / 2},
$$

where $\Lambda=1+\Lambda_{\tilde{X}, \min } / \Lambda_{h, \max }$, while $\delta_{1}^{\prime}$ can be chosen along the lines of Inequality (29) or

$$
\delta_{1}^{\prime}:=\frac{1}{3 \delta_{0}}\left(8 \delta_{0} K_{X}^{2}+8 \kappa_{1}^{\prime} \sqrt{\delta_{0} / 2} \vee 16 \kappa_{0} K_{g} \vee \kappa_{2} / \sqrt{2}\right)
$$

Proof. Let $\left\{\varepsilon_{i}\right\}_{i=1}^{n}$ be a Rademacher sequence independent of $\left\{\left(x_{i}, z_{i}\right)\right\}_{i=1}^{n}$. By symmetrization (see e.g. van der Vaart and Wellner (1996)), we have

$$
\mathbb{E} \sup _{f \in \mathcal{F}(R)}\left|\|f\|_{n}^{2}-\|f\|^{2}\right| \leq 2 \mathbb{E}\left(\sup _{f \in \mathcal{F}(R)}\left|\frac{1}{n} \sum_{i=1}^{n} f_{i}^{2} \varepsilon_{i}\right|\right) .
$$


Note now that for $X \beta+g \in \mathcal{F}(R)$, by Condition 2.2

$$
\|X \beta\|_{\infty} \leq K_{X}\|\beta\|_{1} \leq K_{X} \frac{R^{2}}{\sqrt{2 / \delta_{0}} \lambda} \leq K_{X} \sqrt{\frac{\delta_{0}}{2}}
$$

where we used the assumption that $R^{2} / \lambda \leq 1$. By the contraction inequality (see Ledoux and Talagrand (1991)), we have

$$
\mathbb{E} \sup _{\beta: X \beta+g \in \mathcal{F}(R)}\left|\frac{1}{n} \sum_{i=1}^{n}(X \beta)_{i}^{2} \varepsilon_{i}\right| \leq \frac{4 K_{X}}{\sqrt{2 / \delta_{0}}} \mathbb{E} \sup _{\beta: X \beta+g \in \mathcal{F}(R)}\left|\frac{1}{n} \sum_{i=1}^{n}(X \beta)_{i} \varepsilon_{i}\right| .
$$

But

$$
\begin{aligned}
\mathbb{E} \sup _{\beta: X \beta+g \in \mathcal{F}(R)}\left|\frac{1}{n} \sum_{i=1}^{n}(X \beta)_{i} \varepsilon_{i}\right| & \leq \mathbb{E} \sup _{\beta: X \beta+g \in \mathcal{F}(R)} \frac{1}{n}\left\|\varepsilon^{T} X\right\|_{\infty}\|\beta\|_{1} \\
& \leq \frac{R^{2}}{\sqrt{2 / \delta_{0}} \lambda} \mathbb{E}\left\|\varepsilon^{T} X\right\|_{\infty} / n \\
& \leq \frac{\lambda_{0}}{\lambda} K_{X} \sqrt{\delta_{0} / 2} R^{2} \\
& \leq\left(\delta_{1}^{\prime} K_{X} \sqrt{\delta_{0} / 2}\right) R^{2}
\end{aligned}
$$

where we invoked the Cauchy-Schwarz inequality for the first step, Lemma 14.14 in Bühlmann and van de Geer (2011) for the second, the approximation $\mathbb{E}\left\|\varepsilon^{T} X\right\|_{\infty} / n \leq \lambda_{0} K_{X}$ in the third one and the assumption $\lambda \geq \lambda_{0} / \delta_{1}^{\prime}$ in the last inequality. Thus

$$
\mathbb{E} \sup _{\beta: X \beta+g \in \mathcal{F}(R)}\left|\frac{1}{n} \sum_{i=1}^{n}(X \beta)_{i}^{2} \varepsilon_{i}\right| \leq 4\left(\frac{\delta_{1}^{\prime} K_{X}^{2}}{2 / \delta_{0}}\right) R^{2}=2 \delta_{0} K_{X}^{2} \delta_{1}^{\prime} R^{2} .
$$

As in the proof of Lemma 6.4, we note that for $X \beta+g \in \mathcal{F}(R)$

$$
\left\|\frac{g}{R / \mu}\right\|^{2} \leq \Lambda^{2} \mu^{2} \leq 1
$$

and

$$
J\left(\frac{\mu g}{R}\right) \leq 1 .
$$

Therefore, by Condition 2.5

$$
\|g\|_{\infty} \leq K_{g} R / \mu
$$


Again by the contraction inequality,

$$
\mathbb{E} \sup _{g: X \beta+g \in \mathcal{F}(R)}\left|\frac{1}{n} \sum_{i=1}^{n} g_{i}^{2} \varepsilon_{i}\right| \leq \frac{4 K_{g} R}{\mu} \mathbb{E} \sup _{g: X \beta+g \in \mathcal{F}(R)}\left|\frac{1}{n} \sum_{i=1}^{n} g_{i} \varepsilon_{i}\right| .
$$

From Lemma 6.4 for $\mu^{2} \geq \kappa_{0} n^{-\frac{2 m}{2 m+1}}$,

$$
\mathbb{E} \sup _{g: X \beta+g \in \mathcal{F}(R)}\left|\frac{1}{n} \sum_{i=1}^{n} g_{i} \varepsilon_{i}\right| \leq \kappa_{0} \frac{R}{\sqrt{n} \mu^{\frac{1}{2 m}}} .
$$

It follows that

$$
\begin{gathered}
\mathbb{E} \sup _{g: X \beta+g \in \mathcal{F}(R)}\left|\frac{1}{n} \sum_{i=1}^{n} g_{i}^{2} \varepsilon_{i}\right| \leq \kappa_{0} \frac{4 K_{g} R}{\mu} \frac{R}{\sqrt{n} \mu^{\frac{1}{2 m}}} \\
=4 \kappa_{0} K_{g} \frac{R^{2}}{\sqrt{n} \mu^{\frac{2 m+1}{2 m}}} .
\end{gathered}
$$

We also have

$$
\begin{gathered}
\mathbb{E} \sup _{\beta, g: X \beta+g \in \mathcal{F}(R)}\left|\frac{1}{n} \sum_{i=1}^{n}(X \beta)_{i} g_{i} \varepsilon_{i}\right| \leq \mathbb{E} \sup _{\beta, g: X \beta+g \in \mathcal{F}(R)}\|\beta\|_{1}\left\|\sum_{i=1}^{n} x_{i} g_{i} \epsilon_{i}\right\|_{\infty} \\
\leq \frac{R^{2}}{\lambda} \sqrt{\delta_{0} / 2} \mathbb{E} \max _{1 \leq j \leq p} \sup _{g: X \beta+g \in \mathcal{F}(R)}\left|\frac{1}{n} \sum_{i=1}^{n} X_{i j} g_{i} \varepsilon_{i}\right| .
\end{gathered}
$$

Similarly as in the proof of Lemma 6.4 we have

$$
\begin{gathered}
\left.\mathcal{H}\left(u,\left\{g X_{j}, g \in \mathcal{G}, j=1, \ldots, p\right\},\|\cdot\|_{\infty}\right) \leq \log \sum_{j=1}^{p} N\left(u, g X_{j}, g \in \mathcal{G}\right\},\|\cdot\|_{\infty}\right) \\
\left.\leq \log p+\max _{j=1, \ldots, p} \log N\left(u, g X_{j}, g \in \mathcal{G}\right\},\|\cdot\|_{\infty}\right) \\
\left.=\log p+\max _{j=1, \ldots, p} \mathcal{H}\left(u, g X_{j}, g \in \mathcal{G}\right\},\|\cdot\|_{\infty}\right) \leq \log p+A\left(\frac{R K_{X}}{u \mu}\right)^{\frac{1}{m}},
\end{gathered}
$$

where in the last step we used that $\left\|g X_{j} \mu /\left(R K_{X}\right)\right\| \leq 1$ and conditions 2.2 and 2.4 .

In view of previous results, with help of Lemma 6.4 we have that for $\mu^{2} \geq$ $\kappa_{0} n^{-\frac{2 m}{2 m+1}}$, by Dudley's inequality

$$
\mathbb{E} \max _{1 \leq j \leq p} \sup _{g: X \beta+g \in \mathcal{F}(R)}\left|\frac{1}{n} \sum_{i=1}^{n} X_{i j} g_{i} \varepsilon_{i}\right|
$$




$$
\begin{gathered}
\leq \mathbb{E} \frac{C}{\sqrt{n}} \int_{0}^{R_{n}}\left(\mathcal{H}\left(u,\left\{g X_{j}, g \in \mathcal{G}, j=1, \ldots, p\right\},\|\cdot\|_{\infty}\right)\right)^{\frac{1}{2}} d u \\
\leq \frac{C}{\sqrt{n}} \mathbb{E} \sqrt{\log p} R_{n}+\frac{K_{X}^{\frac{1}{2 m}}}{\sqrt{n}} \mathbb{E} G^{-1}\left(R_{n}^{2}\right) \\
\leq \frac{C \sqrt{8 K_{g} \kappa_{0} \delta_{1}+\Lambda^{2}}}{\sqrt{2}} \lambda_{0} R+K_{X}^{\frac{1}{2 m}} \kappa_{0} \frac{R}{\sqrt{n} \mu^{\frac{1}{2 m}}}
\end{gathered}
$$

In the last steps we used Remark 6.1, Lemma 6.4 and the definition of $\lambda_{0}$.

Hence for, $\mu^{2} \geq\left(\kappa_{0} \vee \kappa_{1}^{\prime} \vee\left(\delta_{1}^{\prime}\right) \frac{-4 m}{2 m+1}\right) n^{-\frac{2 m}{2 m+1}}, \lambda \leq \lambda_{0} / \delta_{1}^{\prime}$ and $\mu \leq R$

$$
\begin{gathered}
\mathbb{E} \sup _{\beta, g: X \beta+g \in \mathcal{F}(R)}\left|\frac{1}{n} \sum_{i=1}^{n}(X \beta)_{i} g_{i} \varepsilon_{i}\right| \\
\leq \frac{R^{2}}{\sqrt{2 / \delta_{0}} \lambda}\left[\frac{C \sqrt{8 K_{g} \kappa_{0} \delta_{1}+\Lambda^{2}}}{\sqrt{2}} \lambda_{0} R+K_{X}^{\frac{1}{2 m}} \kappa_{0} \frac{R}{\sqrt{n} \mu^{\frac{1}{2 m}}}\right] \\
\leq \sqrt{\frac{\delta_{0}}{2}} R^{2} \delta_{1}^{\prime}\left(\frac{C \sqrt{8 K_{g} \kappa_{0} \delta_{1}+\Lambda^{2}}}{\sqrt{2}}+\kappa_{0} K_{X}^{\frac{1}{2 m}} \sqrt{\delta_{0} / 2}\right) \\
=\kappa_{1}^{\prime} \sqrt{\frac{\delta_{0}}{2}} R^{2} \delta_{1}^{\prime}
\end{gathered}
$$

Resuming we have:

$$
\mathbb{E} \sup _{\beta, g: X \beta+g \in \mathcal{F}(R)}\left|\frac{1}{n} \sum_{i=1}^{n}(X \beta)_{i} g_{i} \varepsilon_{i}\right| \leq 2 \kappa_{1}^{\prime} R^{2} \sqrt{\delta_{0} / 2} \delta_{1}^{\prime}
$$

Then, by symmetrization (Corollary 3.4 in van de Geer (2000), van der Vaart and Wellner (1996),

$$
\begin{array}{r}
\mathbb{E} \sup _{f \in \mathcal{F}(R)}\left|\|f\|_{n}^{2}-\|f\|^{2}\right| \leq 2 \mathbb{E} \sup _{f \in \mathcal{F}(R)} \frac{1}{n}\left|\sum_{i=1}^{n} f_{i}^{2} \varepsilon_{i}\right| \\
=2 \mathbb{E} \sup _{f \in \mathcal{F}(R)}\left|\sum_{i=1}^{n}\left(\left(X_{\beta}\right)_{i}^{2} \varepsilon_{i}+2\left(X_{\beta}\right)_{i} g_{i} \varepsilon_{i}+g_{i}^{2} \varepsilon_{i}\right)\right| \\
\leq 4 \delta_{0} K_{X}^{2} \delta_{1}^{\prime} R^{2}+8 \kappa_{0} K_{g} \delta_{1} R^{2}+8 \kappa_{1}^{\prime} \sqrt{\delta_{0} / 2} \delta_{1}^{\prime} R^{2}:=\mathbf{E} .
\end{array}
$$

We moreover have for any $f=X \beta+g$ in $\mathcal{F}(R)$, and for $\kappa_{2}:=K_{X} \delta_{0} / 2+K_{g}$, $\|f\|_{\infty} \leq\|X \beta\|_{\infty}+\|g\|_{\infty} \leq K_{X}\|\beta\|_{1}+\|g\|_{\infty} \leq \kappa_{2} R / \mu$ and $\|f\| \leq R$. Hence, $\left\|f^{2}\right\| \leq\|f\|_{\infty}\|f\| \leq \kappa_{2} R^{2} / \mu$. 
Therefore, for all $t>0$, by Massart's inequality Massart (2000), (see also Corollary 14.2 in Bühlmann and van de Geer (2011)), using the approximation $\kappa_{2} R^{2} / \mu(2 t)^{1 / 2} \cdot\left(1+2 \mathbf{E} / R^{2}\right)^{1 / 2} \leq \mathbf{E}+t \kappa_{2}^{2} R^{2} / \mu^{2}+(2 t)^{1 / 2} R^{2} \kappa_{2} / \mu$,

$$
\begin{aligned}
\mathbb{P}\left(\sup _{f \in \mathcal{F}(R)}\left|\|f\|_{n}^{2}-\|f\|^{2}\right|\right. & \left.\geq 2 \mathbf{E}+\kappa_{2} \frac{R^{2}}{\mu} \sqrt{\frac{2 t}{n}}+\frac{4}{3} \kappa_{2}^{2} \frac{R^{2}}{\mu^{2}} \frac{t}{n}\right) \\
\leq & \exp [-t] .
\end{aligned}
$$

We now take

$$
t:=n\left(\delta_{1}^{\prime \prime}\right)^{2} \mu^{2}
$$

to find

$$
\begin{gathered}
\mathbb{P}\left(\sup _{f \in \mathcal{F}(R)}\left|\|f\|_{n}^{2}-\|f\|^{2}\right| \geq 2 \mathbf{E}+\delta_{1}^{\prime \prime} \kappa_{2} R^{2} \sqrt{2}+\frac{4}{3} \kappa_{2}^{2}\left(\delta_{1}^{\prime \prime}\right)^{2} R^{2}\right) \\
\leq \exp \left[-n\left(\delta_{1}^{\prime \prime}\right)^{2} \mu^{2}\right] .
\end{gathered}
$$

Recall that $\mathbf{E}$ is defined in 28 . Take now $\delta_{1}, \delta_{1}^{\prime}, \delta_{1}^{\prime \prime}$ small enough, that

$$
8 \delta_{0} K_{X}^{2} \delta_{1}^{\prime}+16 \kappa_{0} K_{g} \delta_{1}+16 \kappa_{1}^{\prime} \sqrt{\delta_{0} / 2} \delta_{1}^{\prime}+\delta_{1}^{\prime \prime} \kappa_{2} \sqrt{8}+\frac{8}{3} \kappa_{2}^{2}\left(\delta_{1}^{\prime \prime}\right)^{2} \leq \delta_{0} .
$$

So we have:

$$
\mathbb{P}\left(\sup _{f \in \mathcal{F}(R)}\left|\|f\|_{n}^{2}-\|f\|^{2}\right| \geq \delta_{0} R^{2}\right) \leq \exp \left[-n\left(\delta_{1}^{\prime \prime}\right)^{2} \mu^{2}\right] .
$$

A possible choice for $\delta_{1}, \delta_{1}^{\prime}, \delta_{1}^{\prime \prime}$ respecting (19), is

$$
\delta_{1}^{\prime}:=\delta_{1}^{\prime \prime}:=\frac{\delta_{0}}{3}\left(\left(8 \delta_{0} K_{X}^{2}+16 \kappa_{1}^{\prime} \sqrt{\delta_{0} / 2} \vee 16 \kappa_{0} K_{g}\right) \vee\left(4.6 \kappa_{2}\right)\right)^{-1},
$$

and

$$
\delta_{1}:=\min \left\{\delta_{1}^{\prime},(19)\right\}
$$

\subsection{The set $\mathcal{T}_{2}\left(\delta_{0}, R\right)$}

Lemma 6.6. Assume Conditions 2.1, 2.2, 2.3, 2.4 and 2.5, that $\mu^{2} \leq R^{2}$, (7) and $R^{2} \leq \lambda \leq 1$. 
Then for constant $\delta_{2}$ depending only on $K_{X}, K_{g}, A, m$, for $\delta_{2} \lambda \geq \lambda_{0} R / \mu$, $\lambda_{0}=\sqrt{2 \log (2 p) / n}$ and $\mu^{2} \geq \kappa_{0} \vee \kappa_{1}^{\prime} n^{-\frac{2 m}{2 m+1}}$, we have

$$
\sup _{f \in \mathcal{F}(R)}\left|E^{T} f / n\right| \leq \delta_{0} R^{2}
$$

with probability at least $1-2 \exp \left[-n \delta_{2}^{2} \mu^{2}\right]$

Proof. For $X$ fixed we have

$$
E^{T} X_{j} \sim \mathcal{N}\left(0,\left\|X_{j}\right\|_{2}^{2}\right)
$$

and consequently

$$
\mathbb{E} \max _{1 \leq j \leq p} E^{T} X_{j} \leq \max _{1 \leq j \leq p}\left(\sum_{i=1}^{n} X_{i j}^{2}\right)^{\frac{1}{2}} \sqrt{2 \log p} \leq K_{X} \sqrt{2 \log p} \sqrt{n}
$$

We continue as in the proof of Lemma 6.5, but now with the Rademacher sequence replaced by the Gaussian errors $\left\{e_{i}\right\}$, and conditionally on $X$. Then we obtain

$$
\begin{gathered}
\mathbb{E}\left(\sup _{\beta: X \beta+g \in \mathcal{F}(R)} \mid \frac{1}{n} \sum_{i=1}^{n}(X \beta)_{i} e_{i} \| X\right) \leq \frac{R^{2}}{\sqrt{2 / \delta_{0}} \lambda} \mathbb{E}\left(\left\|E^{T} X\right\|_{\infty} \mid X\right) / n \\
\leq \frac{\lambda_{0} K_{X}}{\sqrt{2 / \delta_{0}} \lambda} R^{2} \leq \frac{\delta_{2} K_{X}}{\sqrt{2 / \delta_{0}}} R \mu \leq \frac{\delta_{2} K_{X}}{\sqrt{2 / \delta_{0}}} R^{2}
\end{gathered}
$$

where we used (32), the definition of $\lambda_{0}$ and Assumption (6). Moreover, conditionally on $Z$

$$
\mathbb{E}\left(\sup _{g: X \beta+g \in \mathcal{F}(R)} \mid \frac{1}{n} \sum_{i=1}^{n} g_{i} e_{i} \| Z\right)=\mathbb{E}\left(\sup _{g: X \beta+g \in \mathcal{F}(R)} \mid \frac{1}{n} \sum_{i=1}^{n} g_{i} e_{i} \epsilon_{i} \| Z\right)
$$

Notice that $\|g e\|=\|g\| \cdot\|e\|=\|g\| \leq \Lambda R$, where $\Lambda:=1+\Lambda_{h, \max } / \Lambda_{\tilde{X} \text {,min }}$. Then the above inequality can be further approximated, with help of Lemma 6.4 as follows:

$$
\leq \kappa_{0} \frac{R_{n}^{1-\frac{1}{2 m}}}{\mu^{\frac{2 m+1}{2 m}} \sqrt{n}} R^{\frac{1}{2 m}} \mu
$$


Recall that $R_{n}=\sup _{g: X \beta+g \in \mathcal{F}(R)}\|g\|_{n}$. For $\mu \geq \delta_{2}^{-\frac{2 m}{2 m+1}} n^{-\frac{m}{2 m+1}}$, we obtain

$$
\begin{aligned}
\mathbb{E}\left(\sup _{f \in \mathcal{F}(R)} \frac{\left|E^{T} f\right|}{n}\right) & \leq \mathbb{E}\left(\sup _{g: X \beta+g \in \mathcal{F}(R)} \frac{\left|E^{T} g\right|}{n}\right)+\mathbb{E}\left(\sup _{\beta: X \beta+g \in \mathcal{F}(R)} \frac{\left|E^{T} X \beta\right|}{n}\right) \\
& \leq \kappa_{0} \frac{R_{n}^{1-\frac{1}{2 m}}}{\mu^{\frac{2 m+1}{2 m}} \sqrt{n}} R^{\frac{1}{2 m}} \mu+\delta_{2} K_{X} \sqrt{\delta_{0} / 2} R^{2}
\end{aligned}
$$

Assuming $R_{n} \leq 2 R$ we would have

$$
\mathbb{E}\left(\sup _{f \in \mathcal{F}(R)} \frac{\left|E^{T} f\right|}{n}\right) \leq 2 \kappa_{0} R^{2} \delta_{2}+\delta_{2} K_{X} \sqrt{\delta_{0} / 2} R^{2} .
$$

Define now the event $\mathbb{A}$ as follows:

$$
\mathbb{A}:=\left\{\sup _{f \in \mathcal{F}(R)}\left|\epsilon^{T} f / n\right| \geq \frac{\delta_{2} K_{X}}{\sqrt{2 / \delta_{0}}} R^{2}+2 \kappa_{0} \delta_{2} R^{2}+2 R \sqrt{\frac{8 t}{n}}\right\}
$$

and

$$
\tilde{R}_{n}=\sup _{f \in \mathcal{F}(R)}\|f\|_{n}
$$

Then, by concentration of measure for Gaussian random variables (see Massart (2003)), for all $t>0$,

$$
\begin{gathered}
\mathbb{P}(\mathbb{A})=\mathbb{E}(\mathbb{P}(\mathbb{A} \mid Z))=\mathbb{E}\left(\mathbb{P}\left(\mathbb{A} \wedge \tilde{R}_{n} \leq 2 R \mid Z\right)\right)+\mathbb{E}\left(\mathbb{P}\left(\mathbb{A} \wedge \tilde{R}_{n}>2 R \mid Z\right)\right) \\
\leq \mathbb{E}\left(\mathbb{P}\left(\mathbb{A} \wedge \tilde{R}_{n} \leq 2 R \mid Z\right)\right)+\mathbb{E}\left(\mathbb{P}\left(\tilde{R}_{n}>2 R \mid Z\right)\right) \leq e^{-t}+\mathbb{P}\left(\tilde{R}_{n}>2 R\right) \\
\leq e^{-t}+\mathbb{P}\left(\tilde{R}_{n}^{2}>4 R^{2}\right) \leq e^{-t}+\mathbb{P}\left(\tilde{R}_{n}^{2}-\sup _{f \in \mathcal{F}(R)}\|f\|^{2} \geq 3 R^{2}\right) \\
\leq e^{-t}+\mathbb{P}\left(\sup _{f \in \mathcal{F}(R)}\left|\|f\|_{n}^{2}-\|f\|^{2}\right| \geq 3 R^{2}\right) \\
\leq e^{-t}+\mathbb{P}\left(\sup _{f \in \mathcal{F}(R)}\left|\|f\|_{n}^{2}-\|f\|^{2}\right| \geq \delta_{0} R^{2}\right) \\
\leq e^{-t}+e^{-\delta_{2}^{2} \mu^{2} n}
\end{gathered}
$$

where in the last step we used Lemma 6.5. Choose now:

$$
t:=\delta_{2}^{2} \mu^{2} n
$$


small enough such that

$$
\frac{\delta_{2} K_{X}}{\sqrt{2 / \delta_{0}}} R^{2}+2 \kappa_{0} \delta_{2} R^{2}+2 R \sqrt{\frac{8 t}{n}} \leq \delta_{0} R^{2}
$$

For example:

$$
\delta_{2}:=\delta_{0}\left(K_{X} \sqrt{\delta_{0} / 2}+2 \kappa_{0}+4 \sqrt{\delta_{0}}\right)^{-1} .
$$

Then we have

$$
\mathbb{P}\left(\mathcal{T}_{2}\left(\delta_{0}, R\right)\right) \geq 1-2 e^{-n \delta_{2}^{2} \mu^{2}}
$$

Proof. of Theorem 3.1

We first assume that we are on $\mathcal{T}$, then as direct consequence of Lemma 6.1, all the conclusions of Theorem 3.1 holds. It is now enough to show that the probability of $\mathcal{T}$ is at least as large as required for satisfying the theorem's requests.

Along the lines of (31), 33), and (20), 27), we can now define

$$
\delta_{T}:=\min \left\{\delta_{1}, \delta_{2}\right\}
$$

and

$$
\kappa_{T}:=\max \left\{\kappa_{0}, \kappa_{1}^{\prime}\right\}
$$

Then we have

$$
\begin{gathered}
\mathbb{P}[\mathcal{T}] \geq 1-\mathbb{P}\left[\mathcal{T}_{1}^{c}\right]-\mathbb{P}\left[\mathcal{T}_{2}^{c}\right] \geq 1-\exp \left[-n\left(\delta_{1}^{\prime \prime}\right)^{2} \mu^{2}\right]-2 \exp \left[-n\left(\delta_{2}\right)^{2} \mu^{2}\right] \\
\leq 1-3 \exp \left[-n\left(\delta_{T}\right)^{2} \mu^{2}\right]
\end{gathered}
$$

Proof. of Theorem 3.2

The idea of the proof is that $(\hat{\beta}, \hat{g})$ is obtained by minimising the penalised sum of squares so the "derivative" has to be 0 in $(\hat{\beta}, \hat{g})$. This is the so called KarashKuhn-Tucker- (KKT-) condition. Similar ideas as in the proof of Theorem 3.1 are used in Lemmas 6.7,6.9, which provide useful approximations for finishing the proof. 
Define

$$
\hat{\beta}_{s}^{j}:=\hat{\beta}+s \mathrm{e}_{j}, \quad \hat{g}_{s}^{j}:=\hat{g}-s h^{j},
$$

where $\mathrm{e}_{j}$ is the $j$-th unit vector of the standard basis of $\mathbb{R}^{p}$. Let then $\hat{\tau}$ satisfy $\|\hat{\tau}\|_{\infty} \leq 1$ and $\hat{\tau}^{T} \hat{\beta}=\|\hat{\beta}\|_{1}$. The KKT-condition is given by

$$
\left.\frac{d}{d s}\left(\left\|Y-X \hat{\beta}_{s}^{j}-\hat{g}_{s}^{j}\right\|_{n}^{2}+\lambda\left\|\hat{\beta}_{s}^{j}\right\|_{1}+\mu^{2} J^{2}\left(\hat{g}_{s}^{j}\right)\right)\right|_{s=0}=0
$$

where the "derivative" is to be understood in sense of subdifferential calculus. Differentiating and using the matrix notation we get

$$
-2(Y-X \hat{\beta}-\hat{g})^{T} \tilde{X} / n+\lambda \hat{\tau}-2 \mu^{2} J(\hat{g}, h)=0,
$$

Writing $Y=X \beta^{0}+g^{0}+E$ we have

$$
2\left(X\left(\hat{\beta}-\beta^{0}\right)+\left(\hat{g}-g^{0}\right)-E\right)^{T} \tilde{X} / n+\lambda \hat{\tau}-2 \mu^{2} J(\hat{g}, h)=0
$$

Hence

$$
\begin{aligned}
& 2\left(\hat{\beta}-\beta^{0}\right)^{T} \tilde{X}^{T} \tilde{X} / n+\lambda \hat{\tau}+2\left(\hat{\beta}-\beta^{0}\right)^{T} h^{T} \tilde{X} / n \\
& +2\left(\hat{g}-g^{0}\right)^{T} \tilde{X} / n+2 E^{T} \tilde{X} / n-2 \mu^{2} J(\hat{g}, h)=0 .
\end{aligned}
$$

Multiplying by $\hat{\beta}-\beta^{0}$ we obtain

$$
\begin{aligned}
& 2\left(\hat{\beta}-\beta^{0}\right)^{T} \tilde{X}^{T} \tilde{X}\left(\hat{\beta}-\beta^{0}\right) / n+\lambda\|\hat{\beta}\|-\hat{\tau}^{T} \beta^{0}+2\left(\hat{\beta}-\beta^{0}\right)^{T} h^{T} \tilde{X}\left(\hat{\beta}-\beta^{0}\right) / n \\
& +2\left(\hat{g}-g^{0}\right)^{T} \tilde{X}\left(\hat{\beta}-\beta^{0}\right) / n+2 E^{T} \tilde{X}\left(\hat{\beta}-\beta^{0}\right) / n-2 \mu^{2} J(\hat{g}, h)\left(\hat{\beta}-\beta^{0}\right)=0 .
\end{aligned}
$$

Note that $\hat{\tau} \beta^{0} \leq\left\|\beta^{0}\right\|_{1}$ and hence $\hat{\tau}^{T} \beta^{0}-\left\|\hat{\beta}_{S_{0}}\right\|_{1} \leq\left\|\left(\hat{\beta}-\beta^{0}\right)_{S_{0}}\right\|_{1}$. Therefore, we have

$$
\begin{gathered}
2\left(\hat{\beta}-\beta^{0}\right)^{T} \tilde{X}^{T} \tilde{X}\left(\hat{\beta}-\beta^{0}\right) / n+\lambda\left\|\hat{\beta}_{S_{0}^{c}}\right\|_{1} \\
\leq \lambda\left\|\left(\hat{\beta}-\beta^{0}\right)_{S_{0}}\right\|_{1}+2\left|\left(\hat{\beta}-\beta^{0}\right)^{T} h^{T} \tilde{X}\left(\hat{\beta}-\beta^{0}\right) / n\right| \\
+2\left|\left(\hat{g}-g^{0}\right)^{T} \tilde{X}\left(\hat{\beta}-\beta^{0}\right) / n\right|+2\left|E^{T} \tilde{X}\left(\hat{\beta}-\beta^{0}\right) / n\right|+2\left|\mu^{2} J(\hat{g}, h)\left(\hat{\beta}-\beta^{0}\right)\right| .
\end{gathered}
$$

We approximate separately each term of the right hand side of the inequality. We use Lemmas 6.7 6.9 and similar arguments as in the proof of Lemmas 6.5 6.6 for the approximation:

$$
\begin{aligned}
\leq & \lambda\left\|\left(\hat{\beta}-\beta^{0}\right)_{S_{0}}\right\|_{1}+2\left(4 \kappa_{0} \delta_{1}+K_{X} \sqrt{2} \delta_{3}^{2}+\frac{8 K_{X} \delta_{3}^{2}}{3}\right) R \mu\left\|\hat{\beta}-\beta^{0}\right\|_{1} \\
& +\left(7 \sqrt{2 \delta_{0}} \delta_{T} R^{2}+2 \sqrt{2 \delta_{0}} R^{2}+4 \sqrt{2} K_{X} \sqrt{\frac{2 \log (2 p)}{n}}\right)\left\|\hat{\beta}-\beta^{0}\right\|_{1} .
\end{aligned}
$$


Recall that $R \mu \leq R^{2} \leq \lambda$. Choose now $\delta_{0}, \delta_{1}$ and $\delta_{3}$ small enough that

$$
\begin{gathered}
2\left(4 \kappa_{0} \delta_{1}+K_{X} \sqrt{2} \delta_{3}+\frac{8 K_{X} \delta_{3}^{2}}{3}\right) R \mu+7 \sqrt{2 \delta_{0}} \delta_{T} R^{2}+ \\
+2 \sqrt{2 \delta_{0}} R^{2}+4 \sqrt{2} K_{X} \sqrt{\frac{2 \log (2 p)}{n}} \leq \frac{\lambda}{2}
\end{gathered}
$$

Hence

$$
2\left\|\tilde{X}\left(\hat{\beta}-\beta^{0}\right)\right\|_{n}^{2}+\frac{\lambda}{2}\left\|\hat{\beta}_{S_{0}^{c}}\right\|_{1} \leq \frac{3 \lambda}{2}\left\|\left(\hat{\beta}-\beta^{0}\right)_{S_{0}}\right\|_{1} .
$$

Adding $\lambda\left\|\left(\hat{\beta}-\beta^{0}\right)_{S_{0}}\right\|_{1} / 2$ on both sides of 36 we obtain:

$$
\begin{gathered}
2\left\|\tilde{X}\left(\hat{\beta}-\beta^{0}\right)\right\|_{n}^{2}+\frac{\lambda}{2}\left\|\hat{\beta}-\beta^{0}\right\|_{1} \leq 2 \lambda\left\|\left(\hat{\beta}-\beta^{0}\right)_{S_{0}}\right\|_{1} \\
\leq 2 \lambda \sqrt{s_{0}}\left\|\hat{\beta}-\beta_{0}\right\|_{2} \\
\leq 2 \lambda \sqrt{s_{0}}\left\|\tilde{X}\left(\hat{\beta}-\beta_{0}\right)\right\|_{2} / \Lambda_{\tilde{X}, \min } \\
\leq \frac{\lambda^{2} s_{0}}{\Lambda_{\tilde{X}, \min }^{2}}+\left\|\tilde{X}\left(\hat{\beta}-\beta_{0}\right)\right\|^{2} \\
\leq \frac{\lambda^{2} s_{0}}{\Lambda_{\tilde{X}, \text { min }}^{2}}+\left\|\tilde{X}\left(\hat{\beta}-\beta_{0}\right)\right\|_{n}^{2}+\lambda\left\|\hat{\beta}-\beta^{0}\right\|_{1} / 4,
\end{gathered}
$$

where we apply the third part of Lemma 6.9 , with $20 \sqrt{\delta_{0} / 2} \delta_{T} R^{2} \leq \lambda / 4$. We find

$$
\left\|\tilde{X}\left(\hat{\beta}-\beta^{0}\right)\right\|_{n}^{2}+\frac{\lambda}{4}\left\|\hat{\beta}-\beta_{0}\right\|_{1} \leq \frac{\lambda^{2} s_{0}}{\Lambda_{\tilde{X}, \min }^{2}} .
$$

This with probability at least $1-3 /(2 p)-\exp \left[-n\left(\delta_{3} \mu^{2}\right)\right]-\mathbb{P}\left(\mathcal{T}^{c}\right)$. Lemmas 6.5 and 6.6 conclude the proof.

Lemma 6.7. Assume Conditions 2.1 and 2.2. Then

$$
E^{T} \tilde{X}\left(\hat{\beta}-\beta^{0}\right) / n \leq \sqrt{8} K_{X} \sqrt{\frac{2 \log (2 p)}{n}}\left\|\hat{\beta}-\beta^{0}\right\|_{1}
$$

with probability at least $1-1 / p$

Proof. We have

$$
E^{T} \tilde{X}\left(\hat{\beta}-\beta^{0}\right) / n \leq\left\|E^{T} \tilde{X} / n\right\|_{\infty}\left\|\hat{\beta}-\beta^{0}\right\|_{1} .
$$


For $\tilde{X}$ fixed, $E^{T} \tilde{X}$ is Gaussian. So for all $t>0$ and all $j$

$$
\mathbb{P}\left(\left|E^{T} \tilde{X}_{j} / n\right| \geq \sqrt{\frac{2 t}{n}}\left\|X_{j}\right\|_{n}\right) \leq 2 \exp [-t] .
$$

Hence

$$
\mathbb{P}\left(\left\|E^{T} \tilde{X} / n\right\|_{\infty} \geq \sqrt{\frac{2(t+\log (p))}{n}} \max _{1 \leq j \leq p}\left\|X_{j}\right\|_{n}\right) \leq 2 \exp [-t] .
$$

Moreover, $\max _{1 \leq j \leq p}\left\|\tilde{X}_{j}\right\|_{n} \leq\|\tilde{X}\|_{\infty} \leq 2 K_{X}$. Now take $t=\log (2 p)$.

Lemma 6.8. Assume Conditions 2.3, 2.6, (4) and (5). Then on $\mathcal{T}$

$$
\mu^{2} J(\hat{g}, h)\left(\hat{\beta}-\beta^{0}\right) \leq \sqrt{2 \delta_{0}} R^{2}\left\|\hat{\beta}-\beta^{0}\right\|_{1}
$$

Proof. Similarly to the previous lemma we have

$$
\mu^{2} J(\hat{g}, h)\left(\hat{\beta}-\beta^{0}\right) \leq \max _{j \in 1, \ldots, p} \mu^{2} J\left(\hat{g}, h_{j}\right)\left\|\hat{\beta}-\beta^{0}\right\|_{1}
$$

Hence, by triangle inequality and Lemma 6.1.

$$
\max _{j \in 1, \ldots, p}\left|J\left(\hat{g}, h_{j}\right)\right| \leq J_{h} J(\hat{g}) \leq \frac{R}{\mu} J_{h}+J_{h} J\left(g_{0}\right)
$$

and

$$
\max _{j \in 1, \ldots, p} \mu^{2}\left|J\left(\hat{g}, h_{j}\right)\right| \leq \sqrt{\delta_{0} / 2} R^{2}
$$

Lemma 6.9. Assume the conditions of Theorem 3.1. Then for all $\delta_{3}>0$, with $\delta_{3} \mu \geq \sqrt{\log p / n}$ except for a set of probability at most $\exp \left[-n\left(\delta_{3}^{2} \mu\right)^{2}\right]$, one has

$$
\left|\frac{1}{n} \sum_{i=1}^{n} g_{i} \tilde{x}_{i} \beta\right| \leq\left(4 \kappa_{0} \delta_{1}+K_{X} \sqrt{2} \delta_{3}^{2}+\frac{8 K_{X} \delta_{3}}{3}\right) R \mu\|\beta\|_{1}
$$

uniformly in $(\beta, g) \in \mathcal{F}(R)$. Furthermore, except for a set with probability at most $1 /(2 p)$, and

$$
\left|\frac{1}{n} \sum_{i=1}^{n}(h \beta)_{i}(\tilde{X} \beta)_{i}\right| \leq 7 \sqrt{\delta_{0} / 2} \delta_{T} R^{2}\|\beta\|_{1}
$$

uniformly in $(\beta, g) \in \mathcal{F}(R)$. 
Finally, also up to a set with probability at most $1 /(2 p)$,

$$
\left|\|\tilde{X} \beta\|_{n}^{2}-\|\tilde{X} \beta\|^{2}\right| \leq 20 \sqrt{\delta_{0} / 2} \delta_{T} R^{2}\|\beta\|_{1} .
$$

uniformly in $(\beta, g) \in \mathcal{F}(R)$.

Proof. As in Lemma 6.4 (applying a symmetrisation step), since by assumption $\mu^{2} \geq \delta_{1}^{-\frac{4 m}{2 m+1}} n^{-\frac{42 m}{2 m+1}}$, we have for all $j$,

$$
\mathbb{E} \sup _{g: X \beta+g \in \mathcal{F}(R)}\left|\frac{1}{n} \sum_{i=1}^{n} g_{i} \tilde{X}_{i j}\right| \leq 4 \kappa_{0} \delta_{1} K_{X} R \mu .
$$

By Massart's inequality, for all $t>0$, and all $j$

$\mathbb{P}\left(\sup _{g: \quad X \beta+g \in \mathcal{F}(R)}\left|\frac{1}{n} \sum_{i=1}^{n} g_{i} \tilde{X}_{i j}\right| \geq 8 \kappa_{0} \delta_{1} R \mu+K_{X} R \sqrt{2 t / n}+\frac{4 K_{X} R t}{3 \mu n}\right) \leq \exp [-t]$.

It follows that for all $t>0$

$$
\begin{gathered}
\mathbb{P}\left(\sup _{g, j, X \beta+g \in \mathcal{F}(R)}\left|\frac{1}{n} \sum_{i=1}^{n} g_{i} \tilde{X}_{i j}\right|\right. \\
\left.\geq 8 \kappa_{0} \delta_{1} R \mu+K_{X} R \sqrt{\frac{2(t+\log p)}{n}}+\frac{4 K_{X} R(t+\log p)}{3 \mu n}\right) \leq \exp [-t] .
\end{gathered}
$$

Choose $t=n\left(\delta_{3} \mu\right)^{2}$ and use that $\delta_{3} \mu \geq \sqrt{\log p / n}$ to get

$$
\begin{gathered}
\mathbb{P}\left(\sup _{g, j, X \beta+g \in \mathcal{F}(R)}\left|\frac{1}{n} \sum_{i=1}^{n} g_{i} \tilde{X}_{i j}\right| \geq 8 \kappa_{0} \delta_{1} R \mu+K_{X} R \sqrt{2} \delta_{3} \mu+\frac{8 K_{X} R \mu \delta_{3}}{3}\right) \\
\leq \exp \left[-n\left(\delta_{3} \mu\right)^{2}\right] .
\end{gathered}
$$

Now apply

$$
\left|\frac{1}{n} \sum_{i=1}^{n} g_{i} \tilde{x}_{i} \beta\right| \leq \max _{1 \leq j \leq p}\left|\frac{1}{n} \sum_{i=1}^{n} g_{i} \tilde{X}_{i j}\right|\|\beta\|_{1}
$$

For the second result, we use

$$
\mathbb{E} \max _{j, k}\left|\frac{1}{n} \sum_{i=1}^{n} h_{i k} \tilde{X}_{i j}\right| \leq \sqrt{2} \frac{4 \log (2 p)}{n} K_{X}^{2}
$$

by Lemma 14.14 in Bühlmann and van de Geer (2011). Using Massart's inequality (Theorem 14.2 in Bühlmann and van de Geer (2011)), we get for all 
$t>0$,

$$
\mathbb{P}\left(\max _{j, k}\left|\frac{1}{n} \sum_{i=1}^{n} h_{i k} \tilde{X}_{i j}\right| \geq 2 \sqrt{\frac{4 \log (2 p)}{n}} K_{X}^{2}+2 K_{X}^{2} \sqrt{\frac{8 t}{n}}\right) \leq \exp [-t] .
$$

Choosing $t=\log (2 p)$ gives

$$
\mathbb{P}\left(\max _{j, k}\left|\frac{1}{n} \sum_{i=1}^{n} h_{i k} \tilde{X}_{i j}\right| \geq 7 \sqrt{\frac{2 \log (2 p)}{n}} K_{X}^{2}\right) \leq \frac{1}{2 p} .
$$

Now apply that for $\beta$ such that $X \beta+g \in \mathcal{F}(R)$,

$$
\left|\frac{1}{n} \sum_{i=1}^{n}(h \beta)_{i} \tilde{X}_{i j}\right| \leq \sqrt{\delta_{0} / 2} \frac{R^{2}}{\lambda} \max _{1 \leq k \leq p}\left|\frac{1}{n} \sum_{i=1}^{n} h_{i k} \tilde{X}_{i j}\right|
$$

and the bound

$$
\lambda \geq \frac{\sqrt{2 \log (2 p) / n}}{\delta_{T}} .
$$

For the third result, we use the same arguments as for the second one. By Lemma 14.14 in Bühlmann and van de Geer (2011)

$$
\mathbb{E} \max _{j, k}\left|\frac{1}{n} \sum_{i=1}^{n}\left(\tilde{X}_{i k} \tilde{X}_{i j}-\mathbb{E} \tilde{X}_{i k} \tilde{X}_{i j}\right)\right| \leq 4 \sqrt{4 \log (2 p) / n} K_{X}^{2}
$$

and by Massart's inequality for all $t$,

$\mathbb{P}\left(\max _{j, k}\left|\frac{1}{n} \sum_{i=1}^{n}\left(\tilde{X}_{i k} \tilde{X}_{i j}-\mathbb{E} \tilde{X}_{i k} \tilde{X}_{i j}\right)\right| \geq 4 \sqrt{4 \log (2 p) / n} K_{X}^{2}+4 K_{X}^{2} \sqrt{8 t}\right) \leq \exp [-t]$.

So for $t=\log (2 p)$,

$$
\mathbb{P}\left(\max _{j, k}\left|\frac{1}{n} \sum_{i=1}^{n}\left(\tilde{X}_{i k} \tilde{X}_{i j}-\mathbb{E} \tilde{X}_{i k} \tilde{X}_{i j}\right)\right| \geq 20 \sqrt{4 \log (2 p) / n} K_{X}^{2}\right) \leq 1 /(2 p) .
$$

Then again apply Hölder's inequality and $\|\beta\|_{1} \leq \sqrt{\delta_{0} / 2} R^{2} / \lambda$ whenever $(\beta, g) \in$ $\mathcal{F}(R)$. 


\section{Tables with results}

Here the results of the simulations are resumed in tables 2,5, 


\begin{tabular}{|c|c|c|c|c|c|c|c|c|c|c|c|}
\hline \multicolumn{12}{|c|}{ Results for the set Leukemia } \\
\hline$n^{\circ}$ & setting & \multicolumn{4}{|c|}{ Estimation error } & \multicolumn{4}{|c|}{$\begin{array}{l}\text { Prediction error } \\
\end{array}$} & \multicolumn{2}{|c|}{$\overline{\left\|\hat{g}-g^{0}\right\|_{n}}$} \\
\hline & $p, s_{0}, \mathrm{STN}, g_{j}$ & DPI & $\mathrm{DPd}$ & LK & LN & $\mathrm{DPi}$ & $\mathrm{DPd}$ & LK & LN & & DPd \\
\hline$\overline{1}$ & $250,5,2, g_{1}$ & 0.87 & 0.91 & 0.71 & 0.71 & 4.81 & 5.83 & 3.95 & 3.95 & 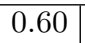 & 0.95 \\
\hline 2 & $250,15,2, g_{1}$ & 1.84 & 1.84 & 1.71 & 1.71 & 18.45 & 18.35 & 16.90 & 16.92 & 1.34 & 1.56 \\
\hline 3 & $1000,5,2, g_{1}$ & 0.97 & 0.99 & 0.83 & 0.83 & 6.56 & 7.16 & 5.28 & 5.32 & 0.64 & 1.03 \\
\hline 4 & 1000,1 & 1.97 & 1.98 & 1.95 & 1.96 & 20.67 & 20.54 & 19.49 & 49.47 & 1.41 & 1.65 \\
\hline 5 & $250,5,8$ & 0.21 & 0.23 & 0.17 & 0.17 & 1.13 & 1.72 & 0.94 & 0.95 & 0.15 & 0.32 \\
\hline 6 & $250,15,8, g_{1}$ & 0.52 & 0.54 & 0.46 & 0.46 & 11.74 & 12.67 & 8.14 & 8.24 & 0.65 & 0.98 \\
\hline 7 & $1000,5,8, g_{1}$ & 0.23 & 0.26 & 0.2 & 0.2 & 1.66 & 2.98 & 1.36 & 1.38 & 0.17 & 0.53 \\
\hline 8 & $1000,15,8, g_{1}$ & 0.77 & 0.78 & 0.85 & 0.85 & 18.92 & 18.78 & 17.2 & 17.25 & 1.02 & 1.32 \\
\hline 9 & $250,5,32, g_{1}$ & 0.06 & 0.07 & 0.05 & 0.05 & 0.23 & 0.40 & 0.19 & 0.19 & 0.04 & 0.10 \\
\hline 10 & 250,1 & 0.24 & 0.24 & 0.17 & 0.17 & 8.54 & 9.85 & 3.61 & 3.72 & 0.47 & 0.80 \\
\hline 11 & 1000 , & 0.07 & 0.08 & 0.06 & 0.06 & 0.38 & 1.18 & 0.30 & 0.30 & 0.05 & 0.26 \\
\hline 12 & $1000,15,32, g_{1}$ & 0.48 & 0.53 & 0.60 & 0.57 & 18.66 & 18.57 & 16.60 & 16.72 & 0.96 & 1.29 \\
\hline 13 & $250,5,2, g_{2}$ & 0.86 & 0.89 & 0.71 & 11.74 & 4.69 & 5.50 & 3.89 & 6.06 & 0.59 & 0.96 \\
\hline 14 & $250,15,2, g_{2}$ & 1.85 & 1.84 & 1.71 & 11.84 & 18.34 & 18.45 & 16.82 & 17.86 & 1.37 & 1.58 \\
\hline 15 & 1000 & 0.96 & 0.97 & 0.83 & 11.74 & 6.16 & 6.65 & \begin{tabular}{ll|}
5.22 \\
\end{tabular} & 7.06 & 0.67 & 1.04 \\
\hline 16 & 1000 , & 1.99 & 1.96 & 1.96 & 11.89 & 20.38 & 20.50 & 19.32 & 19.73 & 1.43 & 1.66 \\
\hline 17 & $250,5,8, g_{2}$ & 0.21 & 0.22 & 0.17 & 11.72 & 0.98 & 1.52 & 0.96 & 5.28 & 0.15 & 0.32 \\
\hline 18 & 250,1 & 0.54 & 0.55 & 0.46 & 11.72 & 11.90 & 12.82 & 8.28 & 15.79 & 0.68 & 1.00 \\
\hline 19 & $1000,5,8, g_{2}$ & 0.23 & 0.27 & 0.20 & 11.71 & 1.52 & 2.85 & 1.40 & 6.37 & 0.18 & 0.56 \\
\hline 20 & $1000,15,8, g_{2}$ & 0.84 & 0.81 & 0.83 & 11.77 & 18.55 & 18.68 & 17.22 & 18.96 & 1.04 & 1.33 \\
\hline 21 & $250,5,32, g_{2}$ & 0.17 & 0.17 & 0.05 & 11.72 & 0.54 & 0.84 & 0.19 & 5.21 & 0.09 & 0.25 \\
\hline 22 & 250, & 0.28 & 0.29 & 0.16 & 11.72 & 8.95 & 10.24 & 3.51 & 15.42 & 0.51 & 0.86 \\
\hline 23 & 1000,5 , & 0.17 & 0.18 & 0.06 & 11.71 & 0.72 & 1.70 & 0.29 & 6.28 & 0.10 & 0.40 \\
\hline 24 & $1000,15,32, g_{2}$ & 0.59 & 0.62 & 0.60 & 11.76 & 18.41 & 118.35 & 16.44 & 18.76 & 1.03 & 1.34 \\
\hline 25 & $250,5,2, g_{3}$ & 0.87 & 0.90 & 0.71 & 3.97 & 4.79 & 5.62 & 3.94 & 7.05 & 0.60 & 0.94 \\
\hline 26 & $250,15,2, g_{3}$ & 1.84 & 1.82 & 1.72 & 4.24 & 18.64 & 18.48 & 16.87 & 18.72 & 1.34 & 1.56 \\
\hline 27 & 1000,5 & 0.97 & 0.99 & 0.83 & 3.99 & 6.44 & 6.95 & 5.27 & 7.76 & 0.66 & 1.02 \\
\hline 28 & $1000,15,2, g_{3}$ & 1.98 & 1.94 & 1.95 & 4.36 & 20.67 & 20.61 & 19.40 & 20.07 & 1.41 & 1.62 \\
\hline 29 & $250,5,8, g_{3}$ & 0.21 & 0.23 & 0.17 & 3.89 & 1.12 & 1.68 & 0.94 & 6.56 & 0.15 & 0.32 \\
\hline 30 & $250,15,8, g_{3}$ & 0.52 & 0.54 & 0.45 & 3.91 & 11.66 & 12.91 & 8.18 & 17.51 & 0.65 & 1.00 \\
\hline 31 & $1000,5,8, g_{3}$ & 0.23 & 0.26 & 0.20 & 3.89 & 1.68 & 2.95 & 1.38 & 7.52 & 0.18 & 0.53 \\
\hline 32 & $1000,15,8, g_{3}$ & 0.76 & 0.80 & 0.83 & 4.02 & 18.88 & 18.79 & 17.27 & 19.76 & 1.02 & 1.34 \\
\hline 33 & $250,5,32, g_{3}$ & 0.08 & 0.08 & 0.05 & 3.88 & 0.26 & 0.48 & 0.19 & 6.62 & 0.05 & 0.13 \\
\hline 34 & $250,15,32, g_{3}$ & 0.24 & 0.25 & 0.17 & 3.89 & 8.52 & 10.05 & 3.57 & 17.36 & 0.47 & 0.81 \\
\hline 35 & $1000,5,32, g_{3}$ & 0.08 & 0.10 & 0.06 & 3.89 & 0.43 & 1.32 & 0.29 & 7.53 & 0.06 & 0.29 \\
\hline 36 & $1000,15,32, g_{3}$ & 0.53 & 0.53 & 0.58 & 4.02 & 18.39 & 18.64 & 16.55 & 19.67 & 0.98 & 1.27 \\
\hline
\end{tabular}

Table 2: The results of the pseudo real data study with the dataset Leukemia are presented here. For the 36 different designs $(N=1, \ldots, 36)$ the performance of Lasso with (LK) and without (LN) prior knowledge on the nuisance function are compared with our estimator in both the independent (DPi) and dependent (DPd) case. Prediction and estimation error for $\hat{\beta}$ and $\hat{g}$ are given. The results are based on 1000 replicates for each design. 


\begin{tabular}{|c|c|c|c|c|c|c|c|c|c|}
\hline \multicolumn{10}{|c|}{ Results for the set Leukemia } \\
\hline$n^{\circ}$ & setting & \multicolumn{4}{|c|}{ TPR } & \multicolumn{4}{|c|}{ FPR } \\
\hline$N$ & $p, s_{0}, \mathrm{STN}, g_{i}$ & DPi & DPd & LK & $\mathrm{LN}$ & DPi & DPd & LK & $\mathrm{LN}$ \\
\hline 1 & $250,5,2, g_{1}$ & 97.9 & 84.0 & $\overline{998.8}$ & 98.8 & 11.4 & 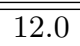 & 9.8 & 9.7 \\
\hline 2 & $250,15,2, g_{1}$ & 56.7 & 51.9 & 65.1 & 64.6 & 13.2 & 12.6 & 12.8 & 12.7 \\
\hline 3 & $1000,5,2, g_{1}$ & 88.8 & 68.8 & 93.9 & 93.5 & 4.0 & 3.9 & 3.3 & 3.3 \\
\hline 4 & $1000,15,2, g_{1}$ & 30.3 & 27.7 & 35.4 & 34.9 & 3.7 & 3.6 & 3.4 & 3.3 \\
\hline 5 & $250,5,8, g_{1}$ & 99.9 & 99.3 & 100 & 100 & 10.4 & 13.3 & 9.5 & 9.3 \\
\hline 6 & $250,15,8, g_{1}$ & 90.5 & 86.0 & 97.5 & 97.5 & 17.4 & 17.4 & 18.5 & 18.5 \\
\hline 7 & $1000,5,8, g_{1}$ & 100 & 95.1 & 100 & 100 & 3.8 & 4.6 & 3.4 & 3.4 \\
\hline 8 & $1000,15,8, g_{1}$ & 50.7 & 47.5 & 61.8 & 61.4 & 4.5 & 4.5 & 4.5 & 4.5 \\
\hline 9 & $250,5,32, g_{1}$ & 100 & 100 & 100 & 100 & 4.0 & 6.5 & 4.8 & 4.7 \\
\hline 10 & $250,15,32, g_{1}$ & 92.6 & 89.9 & 98.8 & 98.7 & 15.1 & 16.4 & 13.7 & 13.7 \\
\hline 11 & $1000,5,32, g_{1}$ & 99.9 & 97.6 & 100 & 100 & 1.8 & 3.0 & 1.9 & 1.9 \\
\hline 12 & $1000,15,32, g_{1}$ & 54.0 & 49.7 & 65.6 & 65.6 & 4.7 & 4.6 & 4.7 & 4.8 \\
\hline 13 & $250,5,2, g_{2}$ & 98.0 & 85.0 & 99.2 & 83.0 & 11.1 & 11.2 & 9.8 & 8.7 \\
\hline 14 & $250,15,2, g_{2}$ & 55.2 & 51.8 & 65.2 & 53.7 & 13.0 & 12.9 & 12.6 & 11.2 \\
\hline 15 & $1000,5,2, g_{2}$ & 88.4 & 68.4 & 94.1 & 65.4 & 3.6 & 3.4 & 3.3 & 2.7 \\
\hline 16 & $1000,15,2, g_{2}$ & 29.8 & 27.8 & 36.1 & 29.1 & 3.5 & 3.6 & 3.3 & 3.0 \\
\hline 17 & $250,5,8, g_{2}$ & 100 & 99.3 & 100 & 91 & 8.2 & 10.4 & 9.8 & 9.5 \\
\hline 18 & $250,15,8, g_{2}$ & 89.7 & 85.1 & 97.1 & 74.2 & 16.8 & 16.9 & 18.5 & 14.3 \\
\hline 19 & $1000,5,8, g_{2}$ & 99.9 & 94.4 & 100 & 77.6 & 2.9 & 3.5 & 3.5 & 3.0 \\
\hline 20 & $1000,15,8, g_{2}$ & 49.3 & 47.0 & 62.5 & 43.2 & 4.2 & 4.3 & 4.6 & 3.8 \\
\hline 21 & $250,5,32, g_{2}$ & 100 & 99.8 & 100 & 91.1 & 1.4 & 3.3 & 4.7 & 9.4 \\
\hline 22 & $250,15,32, g_{3}$ & 93.2 & 89.5 & 98.9 & 76.4 & 14.4 & 15.4 & 13.6 & 14.5 \\
\hline 23 & $1000,5,32, g_{2}$ & 99.9 & 96.6 & 100 & 78.8 & 0.8 & 2.0 & 1.9 & 3.0 \\
\hline 24 & $1000,15,32, g_{2}$ & 51.8 & 48.5 & 66.4 & 44.6 & 4.3 & 4.3 & 4.7 & 3.8 \\
\hline 25 & $250,5,2, g_{3}$ & 97.9 & 85.3 & 99.2 & 68.7 & 11.5 & 11.6 & 9.9 & 8.0 \\
\hline 26 & $250,15,2, g_{3}$ & 57.8 & 53.2 & 65.6 & 46.6 & 13.6 & 13.1 & 12.7 & 10.5 \\
\hline 27 & $1000,5,2, g_{3}$ & 89.3 & 67.9 & 93.8 & 47.7 & 3.9 & 3.7 & 3.3 & 2.4 \\
\hline 28 & $1000,15,2, g_{3}$ & 30.8 & 28.7 & 35.7 & 24.1 & 3.7 & 3.7 & 3.4 & 2.8 \\
\hline 29 & $250,5,8, g_{3}$ & 99.9 & 99.2 & 100 & 76.2 & 0.5 & 13.0 & 9.5 & 8.5 \\
\hline 30 & $250,15,8, g_{3}$ & 90.6 & 85.4 & 97.7 & 59.8 & 17.6 & 17.8 & 18.7 & 12.3 \\
\hline 31 & $1000,5,8, g_{3}$ & 100 & 95.1 & 99.9 & 56.0 & 3.7 & 4.4 & 3.4 & 2.6 \\
\hline 32 & $1000,15,8, g_{3}$ & 51.1 & 47.2 & 62.5 & 32.2 & 4.6 & 4.5 & 4.6 & 3.3 \\
\hline 33 & $250,5,32, g_{3}$ & 100 & 99.9 & 100 & 75.9 & 2.7 & 5.3 & 4.7 & 8.6 \\
\hline 34 & $250,15,32, g_{3}$ & 93.1 & 89.6 & 98.8 & 60.8 & 15.2 & 16.4 & 13.7 & 12.5 \\
\hline 35 & $1000,5,32, g_{3}$ & 99.9 & 97.5 & 100 & 57.5 & 1.4 & 2.8 & 1.9 & 2.6 \\
\hline 36 & $1000,15,32, g_{3}$ & 54.0 & 49.8 & 66.1 & 33.4 & 4.7 & 4.6 & 4.8 & 3.3 \\
\hline
\end{tabular}

Table 3: For the pseudo real data from dataset Leukemia performance of Lasso with (LK) and without (LN) prior knowledge of the nuisance function are compared with our estimator for both independent (DPi) and dependent (DPd) settings. True and false positive rates (TPR and FPR respectively) are given in this table. The results are based on 1000 replicates for each design. 


\begin{tabular}{|c|c|c|c|c|c|c|c|c|c|c|c|}
\hline \multicolumn{12}{|c|}{ Results for the set Prostate } \\
\hline$n^{\circ}$ & setting & \multicolumn{4}{|c|}{ Estimation error } & \multicolumn{4}{|c|}{$\begin{array}{cc}\text { Prediction error } \\
\end{array}$} & \multicolumn{2}{|c|}{$\left\|\hat{g}-g^{0}\right\|_{n}$} \\
\hline$N$ & $p, s_{0}, \mathrm{STN}, g_{j}$ & $\mathrm{DPi}$ & DPd & LK & LN & DPi & $\mathrm{DPd}$ & LK & LN & DPi & $\mathrm{DPd}$ \\
\hline 1 & 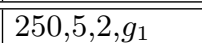 & 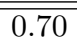 & 0.74 & 0.59 & 0.60 & 4.66 & 5.41 & 4.16 & 4.25 & 0.46 & 0.82 \\
\hline 2 & $250,15,2, g_{1}$ & 1.47 & 1.51 & 1.36 & 1.36 & 16.82 & 17.13 & 16.01 & 16.01 & 0.92 & 1.25 \\
\hline 3 & $1000,5,2, g_{1}$ & 0.79 & 0.82 & 0.69 & 0.70 & 6.11 & 6.53 & 5.37 & 5.41 & 0.49 & 0.96 \\
\hline 4 & $1000,15,2, g_{1}$ & 1.61 & 1.64 & 1.53 & 1.53 & 19.72 & 20.06 & 18.93 & 18.93 & 0.98 & 1.33 \\
\hline 5 & $250,5,8, g_{1}$ & 0.18 & 0.19 & 0.15 & 0.15 & 1.23 & 1.78 & 1.07 & 1.09 & 0.12 & 0.31 \\
\hline 6 & $250,15,8, g_{1}$ & 0.42 & 0.44 & 0.39 & 0.39 & 8.25 & 9.16 & 6.99 & 7.09 & 0.31 & 0.64 \\
\hline 7 & $1000,5,8, g_{1}$ & 0.21 & 0.23 & 0.18 & 0.18 & 20.7 & 3.15 & 1.75 & 1.78 & 0.14 & 0.50 \\
\hline 8 & $1000,15,8, g_{1}$ & 0.54 & 0.55 & 0.51 & 0.51 & 15.59 & 15.86 & 13.91 & 14.02 & 0.49 & 0.93 \\
\hline 9 & $250,5,32, g_{1}$ & 0.06 & 0.06 & 0.05 & 0.05 & 0.33 & 0.57 & 0.28 & 0.29 & 0.03 & 0.15 \\
\hline 10 & $250,15,32, g_{1}$ & 0.21 & 0.21 & 0.20 & 0.20 & 4.70 & 5.46 & 3.47 & 3.54 & 0.16 & 0.46 \\
\hline 11 & $1000,5,32, g_{1}$ & 0.06 & 0.08 & 0.06 & 0.06 & 0.61 & 1.25 & 0.46 & 0.47 & 0.04 & 0.26 \\
\hline 12 & $1000,15,32, g_{1}$ & 0.29 & 0.30 & 0.28 & 0.28 & 13.73 & 14.43 & 11.27 & 11.42 & 0.40 & 0.84 \\
\hline 13 & $250,5,2, g_{2}$ & 0.71 & 0.74 & 0.59 & 11.74 & 4.65 & 5.37 & 4.20 & 6.16 & 0.47 & 0.84 \\
\hline 14 & $250,15,2, g_{2}$ & 1.49 & 1.50 & 1.35 & 11.80 & 16.90 & 17.15 & 15.88 & 17.19 & 0.93 & 1.25 \\
\hline 15 & $1000,5,2, g_{2}$ & 0.80 & 0.81 & 0.70 & 11.75 & 6.06 & 6.58 & 5.48 & 7.07 & 0.49 & 0.93 \\
\hline 16 & $1000,15,2, g_{2}$ & 1.62 & 1.63 & 1.53 & 11.82 & 19.71 & 19.90 & 18.94 & 19.60 & 0.99 & 1.33 \\
\hline 17 & $250,5,8, g_{2}$ & 0.18 & 0.20 & 0.15 & 11.73 & 1.19 & 1.81 & \begin{tabular}{|l|}
1.07 \\
\end{tabular} & 5.51 & 0.12 & 0.32 \\
\hline 18 & $250,15,8, g_{2}$ & 0.42 & 0.43 & 0.38 & 11.72 & 8.27 & 9.33 & 6.92 & 14.92 & 0.31 & 0.66 \\
\hline 19 & $1000,5,8, g_{2}$ & 0.21 & 0.23 & 0.18 & 11.72 & 1.97 & 3.09 & 1.73 & 6.51 & 0.14 & 0.50 \\
\hline 20 & $1000,15,8, g_{2}$ & 0.54 & 0.56 & 0.51 & 11.73 & 15.62 & 15.96 & 13.92 & 18.39 & 0.50 & 0.95 \\
\hline 21 & $250,5,32, g_{2}$ & 0.15 & 0.15 & 0.05 & 11.72 & 0.69 & 1.11 & 0.27 & 5.38 & 0.07 & 0.29 \\
\hline 22 & $250,15,32, g_{3}$ & 0.22 & 0.23 & 0.20 & 11.71 & 5.1 & 5.93 & 3.52 & 14.58 & 0.18 & 0.49 \\
\hline 23 & $1000,5,32, g_{2}$ & 0.15 & 0.16 & 0.06 & 11.72 & 1.07 & 1.89 & 0.44 & 6.48 & 0.08 & 0.41 \\
\hline 24 & $1000,15,32, g_{2}$ & 0.30 & 0.31 & 0.28 & 11.72 & 13.86 & 14.55 & 11.23 & 18.22 & 0.41 & 0.84 \\
\hline 25 & $250,5,2, g_{3}$ & 0.71 & 0.74 & 0.59 & 3.99 & 4.72 & 5.47 & 4.20 & 6.69 & 0.47 & 0.85 \\
\hline 26 & $250,15,2, g_{3}$ & 1.49 & 1.49 & 1.36 & 4.13 & 17.08 & 17.06 & 15.83 & 18.05 & 0.93 & 1.25 \\
\hline 27 & $1000,5,2, g_{3}$ & 0.81 & 0.81 & 0.70 & 3.97 & 6.06 & 6.54 & 5.37 & 7.81 & 0.50 & 0.94 \\
\hline 28 & $1000,15,2, g_{3}$ & 1.63 & 1.62 & 1.53 & 4.2 & 19.86 & 19.93 & 19.00 & 20.05 & 1.00 & 1.33 \\
\hline 29 & $250,5,8, g_{3}$ & 0.18 & 0.19 & 0.15 & 3.94 & 1.24 & 1.86 & 1.09 & 6.38 & 0.12 & 0.32 \\
\hline 30 & $250,15,8, g_{3}$ & 0.42 & 0.44 & 0.39 & 3.91 & 8.22 & 9.31 & 6.98 & 16.78 & 0.31 & 0.66 \\
\hline 31 & $1000,5,8, g_{3}$ & 0.21 & 0.23 & 0.18 & 3.91 & 2.09 & 3.11 & 1.75 & 7.46 & 0.14 & 0.49 \\
\hline 32 & $1000,15,8, g_{3}$ & 0.53 & 0.54 & 0.49 & 3.96 & 15.36 & 15.98 & 13.82 & 19.37 & 0.49 & 0.91 \\
\hline 33 & $250,5,32, g_{3}$ & 0.08 & 0.09 & 0.05 & 3.94 & 0.42 & 0.74 & 0.28 & 6.31 & 0.04 & 0.19 \\
\hline 34 & $250,15,32, g_{3}$ & 0.20 & 0.21 & 0.19 & 3.89 & 4.68 & 5.56 & 3.39 & 16.64 & 0.16 & 0.48 \\
\hline 35 & $1000,5,32, g_{3}$ & 0.09 & 0.10 & 0.06 & 3.91 & 0.74 & 1.45 & 0.48 & 7.47 & 0.05 & 0.30 \\
\hline 36 & $1000,15,32, g_{3}$ & 0.28 & 0.30 & 0.27 & 3.92 & 14.05 & 14.59 & 11.38 & 19.41 & 0.41 & 0.87 \\
\hline
\end{tabular}

Table 4: The results of the pseudo real data study with the dataset Prostate are presented here. For the 36 different designs $(N=1, \ldots, 36)$ the performance of Lasso with (LK) and without (LN) prior knowledge on the nuisance function are compared with our estimator in both the independent (DPi) and dependent (DPd) case. Prediction and estimation error for $\hat{\beta}$ and $\hat{g}$ are given. The results are based on 1000 replicates for each design. 


\begin{tabular}{|c|c|c|c|c|c|c|c|c|c|}
\hline \multicolumn{10}{|c|}{ Results for the set Prostate } \\
\hline$n^{\circ}$ & setting & \multicolumn{4}{|c|}{ TPR } & \multicolumn{4}{|c|}{ FPR } \\
\hline$N$ & $p, s_{0}, \mathrm{STN}, g_{i}$ & DPi & DPd & LK & $\mathrm{LN}$ & DPi & DPd & LK & $\mathrm{LN}$ \\
\hline 1 & $250,5,2, g_{1}$ & 89.8 & 79.3 & $\overline{93.0}$ & 92.7 & 10.8 & $\overline{11.2}$ & 10.3 & $\overline{10.4}$ \\
\hline 2 & $250,15,2, g_{1}$ & 61.8 & 56.9 & 67.2 & 66.1 & 13.6 & 13.3 & 14.0 & 13.8 \\
\hline 3 & $1000,5,2, g_{1}$ & 76.1 & 61.0 & 82.3 & 81.2 & 3.7 & 3.5 & 3.5 & 3.4 \\
\hline 4 & $1000,15,2, g_{1}$ & 39.0 & 34.0 & 43.4 & 42.7 & 4.1 & 4.1 & 4.1 & 4.0 \\
\hline 5 & $250,5,8, g_{1}$ & 99.9 & 98.3 & 99.9 & 99.9 & 11.3 & 13.8 & 10.5 & 10.6 \\
\hline 6 & $250,15,8, g_{1}$ & 94.2 & 91.3 & 96.5 & 96.5 & 18.9 & 19.4 & 19.1 & 19.1 \\
\hline 7 & $1000,5,8, g_{1}$ & 98.4 & 91.9 & 99.2 & 99.2 & 4.4 & 5.2 & 4.2 & 4.2 \\
\hline 8 & $1000,15,8, g_{1}$ & 68.5 & 64.5 & 76.9 & 76.3 & 6.1 & 6.1 & 6.5 & 6.4 \\
\hline 9 & $250,5,32, g_{1}$ & 100 & 99.1 & 100 & 100 & 4.9 & 7.1 & 4.5 & 4.5 \\
\hline 10 & $250,15,32, g_{1}$ & 97.8 & 96.4 & 99.0 & 99.0 & 13.7 & 15.1 & 12.3 & 12.4 \\
\hline 11 & $1000,5,32, g_{1}$ & 99.5 & 97.1 & 99.8 & 99.8 & 2.4 & 3.5 & 2.2 & 2.2 \\
\hline 12 & $1000,15,32, g_{1}$ & 75.5 & 70.9 & 83.9 & 83.3 & 6.2 & 6.3 & 6.4 & 6.4 \\
\hline 13 & $250,5,2, g_{2}$ & 89.9 & 77.7 & 92.5 & 73.5 & 10.7 & 11.1 & 10.5 & 8.7 \\
\hline 14 & $250,15,2, g_{2}$ & 61.6 & 56.3 & 66.6 & 55.1 & 13.7 & 13.4 & 13.8 & 11.8 \\
\hline 15 & $1000,5,2, g_{2}$ & 77.3 & 60.5 & 82.8 & 58.1 & 3.7 & 3.5 & 3.6 & 2.8 \\
\hline 16 & $1000,15,2, g_{2}$ & 38.4 & 35.1 & 43.6 & 34.2 & 4.1 & 4.1 & 4.1 & 3.5 \\
\hline 17 & $250,5,8, g_{2}$ & 99.9 & 98.2 & 99.9 & 80.8 & 10.8 & 13.4 & 10.6 & 9.5 \\
\hline 18 & $250,15,8, g_{2}$ & 94.3 & 91.3 & 96.9 & 72.8 & 19.4 & 20.0 & 19.2 & 15.4 \\
\hline 19 & $1000,5,8, g_{2}$ & 98.5 & 92.7 & 99.3 & 67.6 & 3.9 & 4.8 & 4.2 & 3.1 \\
\hline 20 & $1000,15,8, g_{2}$ & 69.0 & 64.0 & 76.7 & 50.1 & 6.2 & 6.1 & 6.5 & 4.6 \\
\hline 21 & $250,5,32, g_{2}$ & 99.9 & 98.7 & 100 & 81.7 & 2.7 & 4.9 & 4.5 & 9.4 \\
\hline 22 & $250,15,32, g_{3}$ & 97.7 & 96.2 & 98.9 & 74.5 & 13.8 & 15.3 & 12.4 & 15.5 \\
\hline 23 & $1000,5,32, g_{2}$ & 99.5 & 95.6 & 99.9 & 68.9 & 1.5 & 2.6 & 2.2 & 3.2 \\
\hline 24 & $1000,15,32, g_{2}$ & 75.6 & 71.2 & 83.8 & 52.3 & 6.3 & 6.4 & 6.4 & 4.8 \\
\hline 25 & $250,5,2, g_{3}$ & 90.5 & 78.3 & 93.2 & 62.4 & 11.0 & 11.1 & 10.5 & 7.6 \\
\hline 26 & $250,15,2, g_{3}$ & 61.3 & 56.2 & 66.3 & 47.7 & 13.9 & 13.1 & 13.6 & 10.8 \\
\hline 27 & $1000,5,2, g_{3}$ & 76.8 & 60.8 & 82.6 & 45.9 & 3.6 & 3.6 & 3.5 & 2.5 \\
\hline 28 & $1000,15,2, g_{3}$ & 38.3 & 34.0 & 43.6 & 27.8 & 4.1 & 4.1 & 4.1 & 3.2 \\
\hline 29 & $250,5,8, g_{3}$ & 99.8 & 98.3 & 99.9 & 67.1 & 11.4 & 14.3 & 10.9 & 8.1 \\
\hline 30 & $250,15,8, g_{3}$ & 94.1 & 91.3 & 96.5 & 59.8 & 19.2 & 19.7 & 19.1 & 12.9 \\
\hline 31 & $1000,5,8, g_{3}$ & 98.1 & 92.9 & 99.1 & 52.2 & 4.4 & 5.2 & 4.1 & 2.7 \\
\hline 32 & $1000,15,8, g_{3}$ & 70.2 & 64.6 & 77.6 & 37.7 & 6.2 & 6.2 & 6.6 & 3.8 \\
\hline 33 & $250,5,32, g_{3}$ & 99.9 & 99.0 & 100 & 68.2 & 3.6 & 6.2 & 4.5 & 8.0 \\
\hline 34 & $250,15,32, g_{3}$ & 97.8 & 96.4 & 99.1 & 61.2 & 14.0 & 15.3 & 12.4 & 13.2 \\
\hline 35 & $1000,5,32, g_{3}$ & 99.5 & 96.8 & 99.8 & 52.6 & 1.9 & 3.2 & 2.2 & 2.7 \\
\hline 36 & $1000,15,32, g_{3}$ & 75.6 & 70.6 & 83.9 & 39.1 & 6.4 & 6.4 & 6.4 & 3.9 \\
\hline
\end{tabular}

Table 5: For the pseudo real data from dataset Prostate performance of Lasso with (LK) and without (LN) prior knowledge of the nuisance function are compared with our estimator for both independent (DPi) and dependent (DPd) settings. True and false positive rates (TPR and FPR respectively) are given in this table. The results are based on 1000 replicates for each design. 


\section{References}

Bickel, P., Ritov, Y., and Tsybakov, A. (2009). Simultaneous analysis of Lasso and Dantzig selector. Annals of Statistics, 37:1705-1732.

Bühlmann, P. and Mandozzi, J. (2013). High-dimensional variable screening and bias in subsequent inference, with an empirical comparison. Computational Science. To appear.

Bühlmann, P. and van de Geer, S. (2011). Statistics for High-Dimensional Data: Methods, Theory and Applications. Springer.

Dettling, M. (2004). Bagboosting for tumor classification with gene expression data. Bioinformatics, 20(18):3583-3593.

Giné, E. and Koltchinskii, V. (2006). Concentration inequalities and asymptotic results for ratio type empirical processes. The Annals of Probability, 34(3):1143-1216.

Green, P. J., Silverman, B. W., Silverman, B. W., and Silverman, B. W. (1994). Nonparametric regression and generalized linear models: a roughness penalty approach. Chapman \& Hall London.

Green, P. J. and Yandell, B. S. (1985). Semi-parametric generalized linear models. Springer.

Härdle, W. and Liang, H. (2007). Partially linear models. Springer.

Koltchinskii, V. (2009a). The Dantzig selector and sparsity oracle inequalities. Bernoulli, 15:799-828.

Koltchinskii, V. (2009b). Sparsity in penalized empirical risk minimization. Annales de l'Institut Henri Poincaré, Probabilités et Statistiques, 45:7-57.

Koltchinskii, V. (2011). Oracle Inequalities in Empirical Risk Minimization and Sparse Recovery Problems: École dÉté de Probabilités de Saint-Flour XXXVIII-2008, volume 2033. Springer.

Ledoux, M. and Talagrand, M. (1991). Probability in Banach Spaces: Isoperimetry and Processes. Springer Verlag, New York.

Mammen, E. and van de Geer, S. (1997). Penalized quasi-likelihood estimation in partial linear models. Annals of Statistics, 25(3):1014-1035.

Massart, P. (2000). About the constants in Talagrand's concentration inequalities for empirical processes. Annals of Probability, 28:863-884. 
Massart, P. (2003). Concentration inequalities and model selection. Lectures from the 33rd Summer School on Probability Theory held in Saint-Flour, July 6-23, 2003. Lecture Notes in Mathematics.

Tibshirani, R. (1996). Regression shrinkage and selection via the lasso. Journal of the Royal Statistical Society. Series B (Methodological), 58(1):pp. 267-288.

van de Geer, S. (2000). Empirical Processes in M-Estimation. Cambridge University Press.

van de Geer, S. (2007). The deterministic Lasso. In JSM proceedings, 2007, 140. American Statistical Association.

van der Vaart, A. W. and Wellner, J. A. (1996). Weak Convergence and Empirical Processes. Springer Series in Statistics. Springer-Verlag, New York.

Wahba, G. (1990). Spline models for observational data, volume 59. Society for industrial and applied mathematics.

Zou, H. and Hastie, T. (2005). Regularization and variable selection via the elastic net. Journal of the Royal Statistical Society Series B, 67:301-320.

Patric Müller, Seminar für Statistik, Department of Mathematics, ETH Zurich, CH-8092 Zurich, Switzerland.

E-mail: muellepa@stat.math.ethz.ch 